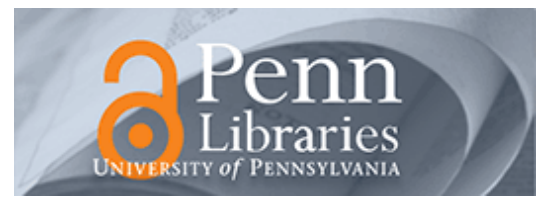

University of Pennsylvania

ScholarlyCommons

4-1-2005

\title{
A Positive Future for Double-Negative Metamaterials
}

\author{
Nader Engheta \\ University of Pennsylvania, engheta@ee.upenn.edu
}

Richard W. Ziolkowski

University of Arizona

Follow this and additional works at: https://repository.upenn.edu/ese_papers

Part of the Materials Science and Engineering Commons

\section{Recommended Citation}

Nader Engheta and Richard W. Ziolkowski, "A Positive Future for Double-Negative Metamaterials", . April 2005.

Copyright 2005 IEEE. Reprinted from IEEE Transactions on Microwave Theory and Techniques, Volume 53, Issue 4, April 2005, pages 1535-1556.

This material is posted here with permission of the IEEE. Such permission of the IEEE does not in any way imply IEEE endorsement of any of the University of Pennsylvania's products or services. Internal or personal use of this material is permitted. However, permission to reprint/republish this material for advertising or promotional purposes or for creating new collective works for resale or redistribution must be obtained from the IEEE by writing to pubs-permissions@ieee.org. By choosing to view this document, you agree to all provisions of the copyright laws protecting it.

This paper is posted at ScholarlyCommons. https://repository.upenn.edu/ese_papers/268

For more information, please contact repository@pobox.upenn.edu. 


\title{
A Positive Future for Double-Negative Metamaterials
}

\begin{abstract}
Metamaterials (MTMs), which are formed by embedding inclusions and material components in host media to achieve composite media that may be engineered to have qualitatively new physically realizable response functions that do not occur or may not be easily available in nature, have raised a great deal of interest in recent years. In this paper, we highlight a large variety of the physical effects associated with double- and single-negative MTMs and some of their very interesting potential applications. The potential ability to engineer materials with desired electric and magnetic properties to achieve unusual physical effects offers a great deal of excitement and promise to the scientific and engineering community. While some of the applications we will discuss have already come to fruition, there are many more yet to be explored.
\end{abstract}

\section{Keywords}

antennas, metamaterials (MTMs), negative index material, negative refraction, resonators, waveguides

\section{Disciplines}

Materials Science and Engineering

\section{Comments}

Copyright 2005 IEEE. Reprinted from IEEE Transactions on Microwave Theory and Techniques, Volume 53, Issue 4, April 2005, pages 1535-1556.

This material is posted here with permission of the IEEE. Such permission of the IEEE does not in any way imply IEEE endorsement of any of the University of Pennsylvania's products or services. Internal or personal use of this material is permitted. However, permission to reprint/republish this material for advertising or promotional purposes or for creating new collective works for resale or redistribution must be obtained from the IEEE by writing to pubs-permissions@ieee.org. By choosing to view this document, you agree to all provisions of the copyright laws protecting it. 


\title{
A Positive Future for Double-Negative Metamaterials
}

\author{
Nader Engheta, Fellow, IEEE, and Richard W. Ziolkowski, Fellow, IEEE
}

Invited Paper

\begin{abstract}
Metamaterials (MTMs), which are formed by embedding inclusions and material components in host media to achieve composite media that may be engineered to have qualitatively new physically realizable response functions that do not occur or may not be easily available in nature, have raised a great deal of interest in recent years. In this paper, we highlight a large variety of the physical effects associated with double- and single-negative MTMs and some of their very interesting potential applications. The potential ability to engineer materials with desired electric and magnetic properties to achieve unusual physical effects offers a great deal of excitement and promise to the scientific and engineering community. While some of the applications we will discuss have already come to fruition, there are many more yet to be explored.
\end{abstract}

Index Terms-Antennas, metamaterials (MTMs), negative index material, negative refraction, resonators, waveguides.

\section{INTRODUCTION}

$\mathbf{O}$ VER 30 years ago, Veselago theoretically considered a homogeneous isotropic electromagnetic material in which both permittivity and permeability were assumed to have negative real values, and he studied uniform plane-wave propagation in such a material, which he referred to as "left-handed (LH)" medium [1], [2]. In such a medium, he concluded, the direction of the Poynting vector of a monochromatic plane wave is opposite to that of its phase velocity, suggesting that this isotropic medium supports backward-wave propagation and its refractive index can be regarded negative. Since such materials were not available until recently, the interesting concept of negative refraction, and its various electromagnetic and optical consequences, suggested by Veselago had received little attention. This was until Smith et al. [5], University of California at San Diego, La Jolla, inspired by the work of Pendry et al. [3], [4] constructed a composite "medium" in the microwave regime by arranging periodic arrays of small metallic wires and split-ring resonators [5]-[8] and demonstrated the anomalous refraction at the boundary of this medium, which is the result of negative refraction in this artificial medium [8]. Since then, many aspects of this class and other related types of artificial materials, now termed metamaterials (MTMs), are being investigated by several groups worldwide, and various ideas and suggestions for potential applications of these media have been mentioned (e.g., [9]-[115]). This has led

Manuscript received July 11, 2004; revised November 8, 2004.

N. Engheta is with the Department of Electrical and Systems Engineering, University of Pennsylvania, Philadelphia, PA 19104-6314 USA (e-mail: Engheta@ee.upenn.edu).

R. W. Ziolkowski is with the Department of Electrical and Computer Engineering, University of Arizona, Tucson, AZ 85721 USA (e-mail: ziolkowski@ece.arizona.edu).

Digital Object Identifier 10.1109/TMTT.2005.845188 to a renewed interest in using fabricated structures to develop composite MTMs that have new physically realizable response functions that do not occur, or may not be readily available, in nature. Among these recent examples of engineered materials, one can mention double-negative (DNG) materials [12], [13] (also known as LH medium [1], negative-index materials (NIMs) [14], [15], backward-wave media (BW) [16], and negative-phase-velocity (NPV) media [17], [18] to name a few); electromagnetic bandgap (EBG) structured materials, and complex surfaces such as high-impedance ground planes and artificial magnetic conductors (AMCs). The new response functions of these MTMs are often generated by artificially fabricated inhomogeneities embedded in host media (volumetric or three-dimensional (3-D) MTMs) or connected to or embedded on host surfaces (planar or two-dimensional (2-D) MTMs).

It is important to point out that the history of artificial materials appears to date back to the late part of the 19th Century when Bose published his work in 1898 on the rotation of the plane of polarization by man-made twisted structures, which were indeed artificial chiral structures by today's definition [19]. Lindman in 1914 studied artificial chiral media formed by a collection of randomly oriented small wire helices [20]. Afterwards, there were several other investigators in the first half of the 20th Century who studied various man-made materials. In the 1950 s and 1960s, artificial dielectrics were explored for lightweight microwave antenna lenses, such as the work of Kock [21]. The 'bed of nails' wire grid medium was used in the early 1960s to simulate wave propagation in plasmas [22]. The interest in artificial chiral materials was resurrected in the 1980s and 1990s (see, e.g., [23]) and they were investigated for various potential device and component applications such as microwave radar absorbers.

Although the majority of the research related to MTMs reported in the recent literature has been concentrated on electromagnetic properties of DNG (LH, NIM. BW, NPV) media, it is worth noting that single-negative (SNG) materials in which only one of the material parameters, not both, has a negative real value may also possess interesting properties when they are juxtaposed in a complementary manner. These media include the only epsilon-negative (ENG) media, such as plasmonic materials like noble metals (silver, gold, etc.) in the visible and infrared (IR) regimes, and the only mu-negative (MNG) media. It has been shown that suitably arranged SNG media may exhibit exciting properties, which may lead to the design of interesting future devices and components (see, e.g., [24]-[27]).

In this paper, we provide an overview of some of the unusual characteristics of DNG MTMs and review some of their exciting potential applications. This paper attempts to address some of the "what-if" questions, namely, if one is able to easily construct 
such DNG media (and there have been ample experimental evidence pointing to construction and engineering aspects of such media), what can one do with them? As such, some of the ideas reviewed in this manuscript are speculative in nature, although they are based on mathematical foundations and are consistent with physical realizability conditions. It is important to point out that currently there are several active research directions in this field, one of which is the research efforts of various groups aimed at the construction and fabrication of 3-D volumetric DNG MTMs by embedding in host media various classes of small inclusions such as wires and split-ring resonators [8], [28]-[34], broadside coupled split-ring resonators [35], capacitively loaded strips and split-ring resonators [13], omega structures [36], [37], and space-filling elements [38] to name a few. Another direction of research effort by several groups is focused on 2-D planar DNG MTMs that utilize circuit and transmission-line implementations. These lumped and distributed circuit element realizations have been used to construct negative-index structures and transmission lines for a variety of applications [39]-[48]. Engineering bandgap structures to control the wave's phase front in order to effectively achieve negative refraction is yet another active area of research relevant to MTMs [49]-[53]. Not all of these topics will be reviewed here since the focus of this paper is on the characteristics and potential applications of DNG MTMs. Furthermore, some of these topics are the subjects of other papers in this TRANSACTIONS.

Although in this paper, we focus on the DNG media, occasionally we will also make remarks on some aspects of SNG media (e.g., plasmonic media) since some of the speculated potential devices that can be formed by DNG media can also be envisioned using SNG materials. This paper cannot obviously include all the potential applications studied by all the groups active in this field, and it only addresses a selected sample of ideas. Thus, we apologize in advance for any omission and oversight in this regard.

A comment about the terminology and notations: among the various possible terminologies for this class of MTMs currently used by various communities, we favor the descriptor DNG for the isotropic case because, in our opinion, it emphasizes the fundamental description of the material. It will be used throughout even though many of the other terms have been equally popular. We use the time-harmonic convention $e^{j \omega t}$ for monochromatic time variations. It is also assumed that the DNG MTMs are lossless at the frequency of interest unless specified otherwise. However, when dissipation is considered, the complex parameters $\varepsilon=\varepsilon_{0}\left(\varepsilon_{r}-j \varepsilon_{i}\right)$ and $\mu=\mu_{0}\left(\mu_{r}-j \mu_{i}\right)$ are used where $\varepsilon_{i}$ and $\mu_{i}$ are nonnegative quantities for passive media. We will also consider any losses to be relatively small, i.e., $\varepsilon_{i} \ll\left|\varepsilon_{r}\right|$ and $\mu_{i} \ll\left|\mu_{r}\right|$. Furthermore, we also simplify the discussion by assuming that the MTMs under discussion are isotropic. Almost all of the realizations of DNG or SNG MTMs to date are by their nature anisotropic or bianisotropic. There is, however, a strong motivation to achieve isotropic properties and this too is under investigation. For instance, the role of anisotropy in the sign of the permittivity and permeability of materials has been investigated in order to achieve certain unconventional features in wave propagation [116].

\section{Negative ReFraction AND CAUSALITY IN DNG MEDIA}

The index of refraction of a DNG MTM has been shown to be negative (e.g., [6], [12], and [39]), and there have now been several theoretical and experimental studies that have been reported confirming this negative index of refraction (NIR) property and applications derived from it such as phase compensation and electrically small resonators [54], negative angles of refraction (e.g., [8], [54]-[59]), sub-wavelength waveguides with lateral dimension below diffraction limits (e.g., [26], [27], [60]-[63]) enhanced focusing (see [46] and [64]), backward wave antennas [44], Čerenkov radiation [65], photon tunneling [66], [67], and enhanced electrically small antennas [68]. These studies rely heavily on the concept that a continuous wave (CW) excitation of a DNG medium leads to a negative refractive index and, hence, to negative or compensated phase terms. Ziolkowski and Heyman thoroughly analyzed this concept mathematically using detailed steps, and have shown that, in DNG media, the refractive index can be negative [12]. One must exercise some care with the definitions of the electromagnetic properties in a DNG medium. When $\varepsilon<0$ and $\mu<0$ in a lossless DNG medium, with the branch-cut choices, as shown in [12], one should write $\sqrt{\varepsilon}=\sqrt{-|\varepsilon|}=-j \sqrt{|\varepsilon|}$ and $\sqrt{\mu}=\sqrt{-|\mu|}=-j \sqrt{|\mu|}$. This leads to the following expressions for the definitions of the wavenumber and the wave impedance, respectively:

$$
k=\omega \sqrt{\varepsilon} \sqrt{\mu}=-\omega \sqrt{|\varepsilon|} \sqrt{|\mu|} \quad \eta=\frac{\sqrt{\mu}}{\sqrt{\varepsilon}}=\frac{\sqrt{|\mu|}}{\sqrt{|\varepsilon|}}
$$

which are needed to properly describe the interaction of a wave with a DNG medium. If the index of refraction of a medium is negative, then the refracted angle, according to Snell's law, should also become "negative." This suggests that the refraction is anomalous, and the refracted angle is on the same side of the interface normal as the incident angle is. This will be clearly shown here later.

Veselago in his 1968 paper mentions certain temporal dispersions for negative permittivity and permeability [1]. As for the causality, we note that if one totally ignores the temporal dispersion in a DNG medium and carefully consider the ramifications of a homogeneous nondispersive DNG medium and the resulting NIR, one will immediately encounter a causality paradox in the time domain, i.e., a nondispersive DNG medium is noncausal. However, a resolution of this issue was uncovered in [69] by taking the dispersion into account in a time-domain study of wave propagation in DNG media. The causality of waves propagating in a dispersive DNG MTM was investigated there both analytically and numerically using the one-dimensional (1-D) electromagnetic plane-wave radiation from a current sheet source in a dispersive DNG medium. In that study, a lossy Drude model of the DNG medium was used, and the solution was generated numerically with the finite-difference time-domain (FDTD) method. The analogous problem in a nondispersive DNG medium was also considered, and it was shown that the solution to this problem is not causal in agreement with similar observations given in [6]. An approximate solution was constructed that combined a causal envelope with a sinusoid, which has the nondispersive NIR properties; it compared favorably with the FDTD results for the dispersive DNG case. It was thus demonstrated that causal results do indeed require the presence of dispersion in DNG media and that the dispersion is responsible for a dynamic reshaping of the pulse to maintain causality. The $\mathrm{CW}$ portions of a modulated pulse (i.e., excluding its leading and trailing edges) do obey all of the NIR effects expected from a 
time-harmonic analysis in a band-limited "nondispersive" DNG medium. Therefore, one can conclude that the $\mathrm{CW}$ analyses of DNG media are credible as long as very narrow bandwidth pulse trains are considered for any practical realizations. This has been the case in all of the experimental results reported to date that we are aware of. Moreover, time delays for the realization of the NIR effects are inherent in the processes dictated by the dispersive nature of the physics governing these media.

Since, in this paper, in addition to analytical descriptions, we present several FDTD simulation results for wave interactions with DNG media, particularly the FDTD representation of negative refraction, here we need to briefly discuss some of the features of the FDTD simulator specific to the DNG structures. It should be emphasized that the use of this purely numerical simulation approach does not involve any choices in defining derived quantities to explain the wave physics, e.g., neither wave vector directions, nor wave speeds are stipulated $a$ priori. In this manner, it has provided a useful approach to studying the wave physics associated with DNG MTMs.

As in [12], [57], [58], and [104], lossy Drude polarization and magnetization models are used to simulate the DNG medium. In the frequency domain, this means the permittivity and permeability are described as

$$
\begin{aligned}
& \varepsilon(\omega)=\varepsilon_{o}\left(1-\frac{\omega_{p e}^{2}}{\omega\left(\omega-j \Gamma_{e}\right)}\right) \\
& \mu(\omega)=\mu_{o}\left(1-\frac{\omega_{p m}^{2}}{\omega\left(\omega-j \Gamma_{m}\right)}\right)
\end{aligned}
$$

where $\omega_{p}$ and $\Gamma$ denote the corresponding plasma and damping frequencies, respectively. Although in some of the analytical and numerical studies, as well as experiments considered by other groups (e.g., [5]-[8], [36], [113], and [114]), the Lorentz model and its derivatives have been used, here the Drude model is preferred for the FDTD simulations for both the permeability and permittivity functions because it provides a much wider bandwidth over which the negative values of the permittivity and permeability can be obtained. This choice is only for numerical convenience, and it does not alter any conclusions derived from such simulations since the negative refraction is observed in either choice. However, choosing the Drude model for the FDTD simulation also implies that the overall simulation time can be significantly shorter, particularly for low-loss media. In other words, the FDTD simulation will take longer to reach a steady state in the corresponding Lorentz model because the resonance region where the permittivity and permeability acquire their negative values would be very narrow in this model. Choice of electric and magnetic currents, polarization and magnetization fields, and the discretization of the computational space for the FDTD simulation are done self-consistently following the conventional FDTD method [119]. The simulation space was truncated with an MTM-based absorbing boundary condition [120], [121]. The FDTD cell size in all cases presented here was $\lambda_{0} / 100$ to minimize the impact of any numerical dispersion on the results.

As an example of numerical simulation of negative refraction, Fig. 1 presents two cases of CW Gaussian wave interaction with DNG slabs when the CW frequency is chosen to be $f_{o}=$ $30 \mathrm{GHz}$. (Needless to say, this choice is arbitrary; the numerical results presented below can be obtained at any frequency with

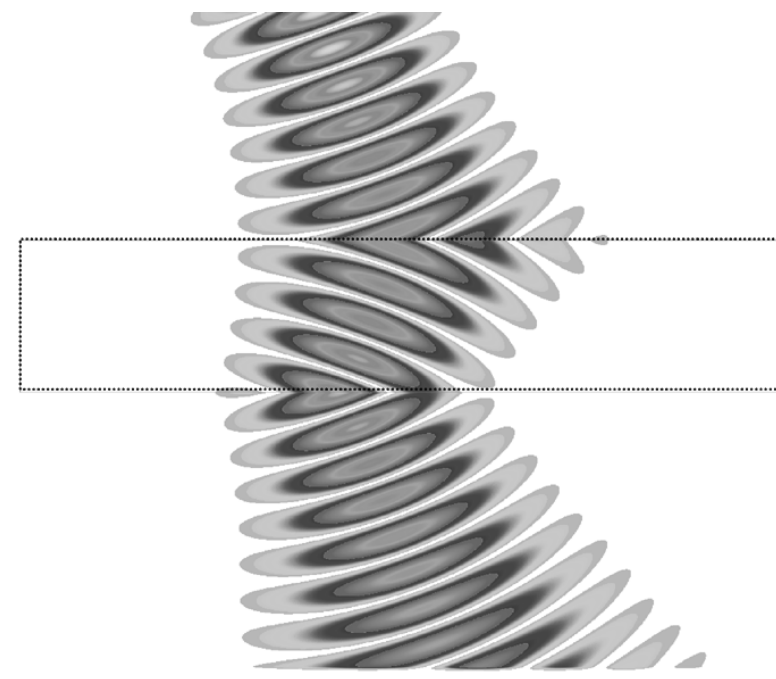

(A)

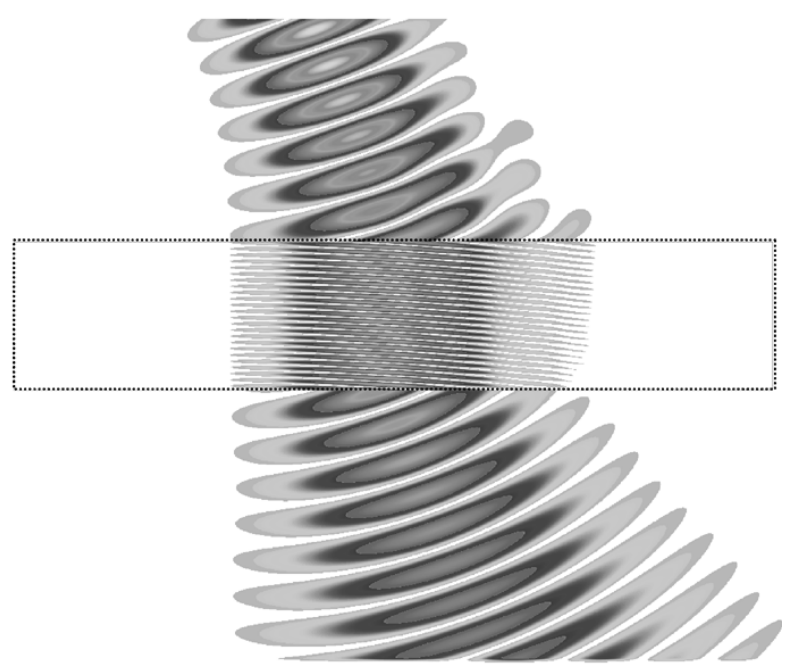

(B)

Fig. 1. FDTD predicted electric-field intensity distribution for the interaction of the CW Gaussian beam that is incident at $20^{\circ}$ to a matched DNG slab having: (A) $n_{\text {real }}\left(\omega_{0}\right) \approx-1$ and $(B) n_{\text {real }}\left(\omega_{0}\right) \approx-6$ with $\omega_{o}=2 \pi \times 3 \times 10^{10} \mathrm{rad} / \mathrm{s}$. A negative angle of refraction equal and opposite to the angle of incidence is clearly observed.

a proper scaling of the parameters.) In order to reduce the effect of reflection and, thus, to observe the negative refraction more clearly, the parameters of these slabs are chosen such that the slab are impedance matched to the free space. Therefore, the electric and magnetic Drude models were selected to be identical, i.e., $\omega_{p e}=\omega_{p m}=\omega_{p}$ and $\Gamma_{e}=\Gamma_{m}=\Gamma$. In all cases, only low-loss values were considered by setting $\Gamma=10^{+8} s^{-1} \ll \omega_{p}$. This means that the index of refraction has the form

$$
\begin{aligned}
n(\omega) & =\sqrt{\frac{\varepsilon(\omega) \mu(\omega)}{\varepsilon_{0} \mu_{0}}} \\
& =1-\frac{\omega_{p}^{2}}{\omega(\omega-j \Gamma)} \\
& =1-\frac{\omega_{p}^{2}}{\omega^{2}+\Gamma^{2}}-j \frac{\Gamma}{\omega} \frac{\omega_{p}^{2}}{\omega^{2}+\Gamma^{2}} \\
& \approx 1-\frac{\omega_{p}^{2}}{\omega^{2}}-j \frac{\Gamma \omega_{p}^{2}}{\omega^{3}} .
\end{aligned}
$$


In Fig. $1(\mathrm{~A})$, the matched DNG slab has $n_{\text {real }}\left(\omega_{0}\right) \approx-1$, when $\omega_{p}=2 \pi \sqrt{2} f_{o}=2.66573 \times 10^{11} \mathrm{rad} / \mathrm{s}$ and, hence, $\Gamma=$ $3.75 \times 10^{-4} \omega_{p}$. For the other matched DNG slab, shown in Fig. $1(\mathrm{~B}), n_{\text {real }}\left(\omega_{0}\right) \approx-6$, for which the model parameters were selected to be $\omega_{p}=2 \pi \sqrt{7} f_{0}=4.98712 \times 10^{11} \mathrm{rad} / \mathrm{s}$ and $\Gamma=2.01 \times 10^{-4} \omega_{p}$.

As can be seen in Fig. 1, the negative angle of refraction is clearly seen in both cases. In panel $(\mathrm{A})$, where $n_{\text {real }}\left(\omega_{0}\right) \approx-1$, the refracted angle is equal and opposite to the angle of incidence, while in panel (B), with $n_{\text {real }}\left(\omega_{0}\right) \approx-6$, the negative angle of refraction is negative, but less than the incident angle. Due to the change in wavelength in the DNG slab, the beam becomes highly compressed along the beam axis. The discontinuities in the derivatives of the fields at the double-positive (DPS)-DNG interfaces (i.e., the so-called "V-shaped" patterns at both interfaces) are clearly seen.

These cases clearly show the presence and effects of the negative angle of refraction realized when an obliquely incident Gaussian beam interacts with a DNG slab. Power flow at the negative angles predicted by Snell's law is confirmed. Fine resolution-in-time movies (not shown here) of the behavior of the electric field in the interaction cases demonstrate that the phase propagation is indeed in the opposite direction to the power flow shown in the figures given here. The FDTD simulation results such as these have numerically confirmed many of the fundamental properties of beam interactions with a DNG medium. We note that there had been some controversy about this negative angle of refraction [14] despite initial experimental verification [8] with 3-D MTM constructs. However, this has already been resolved through the subsequent explanation [15]. More recent planar negative-index transmission line [40], [43] and related planar refractive cone experiments [70], [71] have also more clearly verified this effect.

It is also worth mentioning that Foster's reactance theorem has been shown to be satisfied for the lossless DNG MTMs [72], just as it is for the conventional DPS media.

\section{Phase Compensation IN DNG Media}

One of the interesting features of DNG media is their ability to provide phase compensation or phase conjugation due to their negative index. Consider a slab of conventional lossless DPS material with positive index of refraction $n_{1}$ and thickness $d_{1}$ and a slab of lossless DNG MTM with negative refractive index $-\left|n_{2}\right|$ and thickness $d_{2}$. Although not necessary, but for the sake of simplicity in the argument, we assume that each of these slabs is impedance matched to the outside region (e.g., free space). Let us take a monochromatic uniform plane wave normally incident on this pair of slabs. As this wave propagates through the slab, the phase difference between the exit and entrance faces of the first slab is obviously $n_{1} k_{o} d_{1}$, where $k_{o} \equiv \omega \sqrt{\varepsilon_{o} \mu_{o}}$, while the total phase difference between the front and back faces of this two-layer structure is $\left|n_{1}\right| k_{o} d_{1}-\left|n_{2}\right| k_{o} d_{2}$, implying that whatever phase difference is developed by traversing the first slab, it can be decreased and even compensated by traversing the second slab. If the ratio of $d_{1}$ and $d_{2}$ is chosen to be $d_{1} / d_{2}=\left|n_{2}\right| /\left|n_{1}\right|$ at the given frequency, then the total phase difference between the front and back faces of this two-layer structure will become zero. This means that the DNG slab acts as a phase compensator

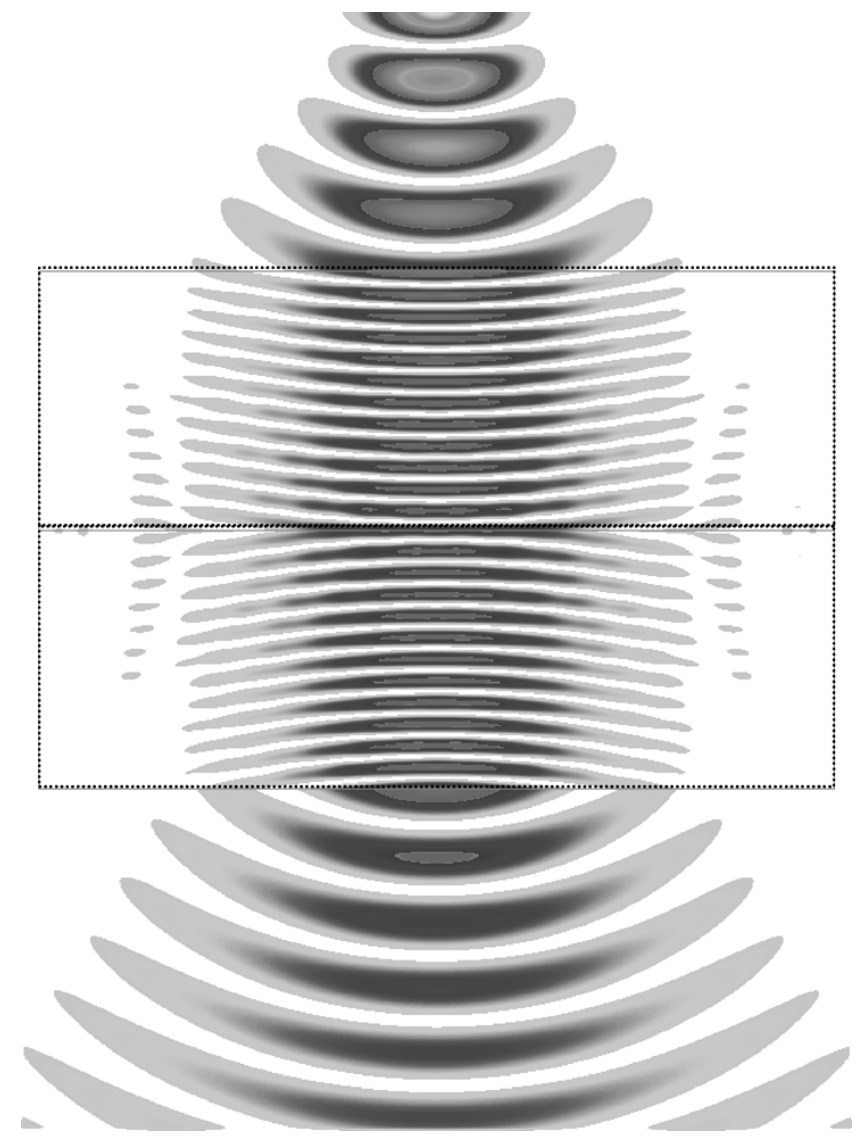

Fig. 2. FDTD predicted electric-field intensity distribution for the phase compensator/beam translator system of a DPS-DNG stacked pair. The Gaussian beam is normally incident on a stack of two slabs, the first being a DPS slab with $n_{\text {real }}(\omega)=+3$ and the second being a DNG slab with $n_{\text {real }}\left(\omega_{0}\right) \approx-3$. The initial beam expansion in the DPS slab is compensated by its refocusing in the DNG slab. The Gaussian beam is translated from the front face of the system to its back face with only a $-0.323-\mathrm{dB}$ attenuation over the $4 \lambda_{0}$ distance.

in this structure [54]. We should note that such phase compensation/conjugation does not depend on the sum of thicknesses $d_{1}+d_{2}$, rather it depends on the ratio of $d_{1}$ and $d_{2}$. Thus, in principle, $d_{1}+d_{2}$ can be any value as long as $d_{1} / d_{2}$ satisfies the above condition. Therefore, even though this two-layer structure is present, the wave traversing this structure would not experience the phase difference. This feature can lead to several interesting ideas in device and component designs, as will be discussed later.

Such phase compensation can also be verified using the FDTD simulation, as shown in Fig. 2. A Gaussian beam is launched toward a pair of DPS and DNG layers, each layer having a thickness of $2 \lambda_{\circ}$. The DPS slab has $n(\omega)=+3$, while the DNG layer has $n_{\text {real }}\left(\omega_{0}\right) \approx-3$. As is evident from Fig. 2, the beam expands in the DPS slab and then refocuses in the DNG slab, and the waist of the intensity of the beam is recovered at the back face. The electric-field intensity could, in principle, be maintained over the total thickness of $4 \lambda_{0}$. There is only a $-0.323 \mathrm{~dB}(7.17 \%)$ reduction in the peak value of the intensity of the beam when it reaches the back face. Moreover, the phase of the beam at the output face of the stack is the same as its value at the entrance face. Therefore, the phase compensator thus translates the Gaussian beam from its front face to its back face with low loss. 
Using multiple matched DPS-DNG stacks, one could produce a phase-compensated time-delayed waveguiding system. Each pair in the stack would act as shown in Fig. 2. Thus, the phase compensation-beam translation effects would occur throughout the entire system. Moreover, by changing the index of any of the DPS-DNG pairs, one changes the speed at which the beam traverses that slab pair. Consequently, one can change the time for the beam to propagate from the entrance face to the exit face of the entire DPS-DNG stack. In this manner, one could realize a volumetric low-loss time-delay line for a Gaussian beam system.

This phase compensation can lead to a wide variety of potential applications that could have a large impact on a number of engineering systems. One such set of applications offers the possibility of having sub-wavelength electrically small cavity resonators and waveguides with lateral dimension below diffraction limits. These ideas are briefly reviewed here. The interested reader is referred to [26], [27], and [54] for further details.

\section{Sub-Wavelength Compact Cavity Resonator USING DNG MEDIA}

Let us take the pair of DPS and DNG layers discussed above and put two perfect reflectors (e.g., two perfectly conducting plates) at the two open surfaces of this bilayer structure, forming a 1-D cavity resonator [see Fig. 3(A)]. It has been shown by Engheta [54] that such a cavity resonator may possess a nonzero mode even when the thickness of the cavity is, in principle, much smaller than the conventional $\lambda / 2$. In that analysis, the dispersion relation was found to be

$$
\frac{n_{\mathrm{DNG}}}{\mu_{\mathrm{DNG}}} \tan \left(n_{\mathrm{DPS}} k_{o} d_{\mathrm{DPS}}\right)+\frac{n_{\mathrm{DPS}}}{\mu_{\mathrm{DPS}}} \tan \left(n_{\mathrm{DNG}} k_{o} d_{\mathrm{DNG}}\right)=0
$$

and since $\varepsilon_{\mathrm{DPS}}>0, \mu_{\mathrm{DPS}}>0, \varepsilon_{\mathrm{DNG}}<0$, and $\mu_{\mathrm{DNG}}<0$, it can be rewritten as

$$
\frac{\tan \left(n_{\mathrm{DPS}} k_{o} d_{\mathrm{DPS}}\right)}{\tan \left(n_{\mathrm{DNG}} k_{o} d_{\mathrm{DNG}}\right)}=\frac{n_{\mathrm{DPS}}\left|\mu_{\mathrm{DNG}}\right|}{n_{\mathrm{DNG}}\left|\mu_{\mathrm{DPS}}\right|} .
$$

(The choice of sign for $n_{\mathrm{DNG}}$ and $n_{\mathrm{DPS}}$ is irrelevant here since either sign does not change this relation.) This relation does not show any direct constraint on the sum of thicknesses of $d_{\mathrm{DPS}}$ and $d_{\mathrm{DNG}}$. Instead, it deals with the ratio of tangent of these thicknesses (with multiplicative constants). This implies that, contrary to a conventional DPS-DPS cavity resonator, here, as $d_{\text {DPS }}$ is reduced, the value of $d_{\text {DNG }}$ can also become smaller in order to satisfy the above dispersion relation (5), and the layers can conceptually be as thin or as thick as otherwise needed as long as relation (5) is satisfied. The total thickness of such a thin cavity $d_{\mathrm{DPS}}+d_{\mathrm{DNG}}$ may turn out to be much smaller than the standard $\lambda / 2$, which, for low-frequency applications, may provide significant miniaturization in cavity resonator designs.

The electric- and magnetic-field expressions for the mode in such a DPS-DNG 1-D cavity have been derived in [54]. Fig. 3(B) presents a sketch of these field distributions in this cavity. As noted in Figs. 1 and 2, and as shown in Fig. 3(B), the tangential electric field possesses a discontinuous first derivative at the boundary between the two

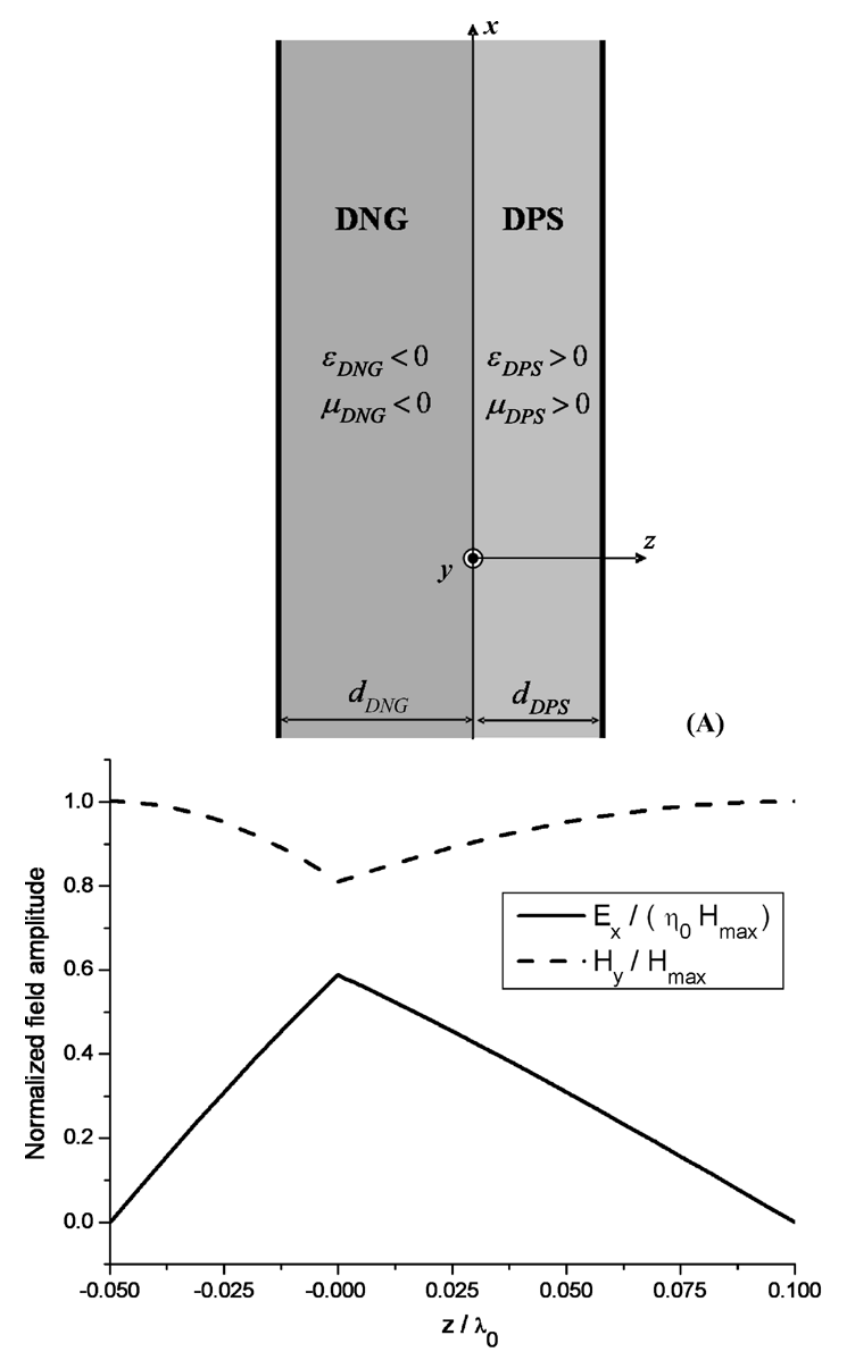

Fig. 3. (A) Sub-wavelength compact cavity resonator formed by a pair of DPS and DNG layers sandwiched between two reflectors. The cavity mode can exist in this structure even when the total thickness may be less than the conventional $\lambda / 2$. (B) Sketch of the normalized magnitude of electric $\left(E_{x}\right)$ and magnetic field $\left(H_{y}\right)$ distribution (with respect to $\eta_{o} H_{y}$ ) as a function of the $z$ coordinate. Here, we take $d_{\mathrm{DPS}} / \lambda_{o}=0.1, d_{\mathrm{DNG}} / \lambda_{o}=0.05, \varepsilon_{\mathrm{DPS}}=\varepsilon_{o}, \mu_{\mathrm{DPS}}=\mu_{o}$, $\varepsilon_{\mathrm{DNG}}=-2 \varepsilon_{o}$, and $\mu_{\mathrm{DNG}}=-2 \mu_{o}$. In this case, the DPS slab occupies the region $0<z / \lambda_{o}<0.1$, while the DNG slab occupies $-0.05<z / \lambda_{o}<0$. From [60].

layers, i.e., $\left(-j \omega \mu_{\mathrm{DPS}}\right)^{-1} \partial_{z} E_{x, \mathrm{DPS}}(z=$ Interface $)=$ $\left(-j \omega \mu_{\mathrm{DNG}}\right)^{-1} \partial_{z} E_{x, \mathrm{DNG}}(z=$ Interface $)$ with $\mu_{\mathrm{DPS}}>0$ and $\mu_{\mathrm{DNG}}<0$. A similar argument can be used for the magnetic-field behavior at this interface.

This idea has also been extended to the cases of cylindrical and spherical cavities filled with a pair of coaxial DPS-DNG layers and concentric DPS-DNG shells, respectively [73], [74]. The corresponding dispersion relations and field distributions have been obtained. As in the case of a 1-D cavity resonator, it has been found that it is possible to have these 2-D and 3-D cavity geometries with the dimension below the conventional cavity size when they are filled with a pair of DPS and DNG layers [73], [74]. An experimental confirmation of the 1-D cavity resonator concept has been recently shown by Hrabar $e t$ $a l$. at a recent symposium [75].

It is worth noting that it is also possible to have such compact cavity resonators using SNG media, such as pairs of ENG and 
MNG layers [26], [27]. In the case of a 1-D ENG-MNG cavity resonator, the dispersion relation takes the form

$$
\begin{aligned}
& \sqrt{\frac{\mu_{\mathrm{ENG}}}{\left|\varepsilon_{\mathrm{ENG}}\right|}} \tanh \left(\omega d_{\mathrm{ENG}} \sqrt{\mu_{\mathrm{ENG}}\left|\varepsilon_{\mathrm{ENG}}\right|}\right) \\
& =\sqrt{\frac{\left|\mu_{\mathrm{MNG}}\right|}{\varepsilon_{\mathrm{MNG}}}} \tanh \left(\omega d_{\mathrm{MNG}} \sqrt{\left|\mu_{\mathrm{MNG}}\right| \varepsilon_{\mathrm{MNG}}}\right) .
\end{aligned}
$$

Such a compact cavity of an ENG-MNG pair can support only one mode at a given frequency. Since this paper deals with the concept of DNG materials and their potential applications, here we do not include the various aspects of the SNG materials and their relevant applications. Further details for the case of ENG-MNG cavities can be found in [26] and [27].

\section{Sub-WaVelength Guided-WaVe Structures With LATERAL DIMENSIONS BELOW DIFFRACTION LIMITS}

The concept of sub-wavelength cavity resonators using DPS-DNG or ENG-MNG layers, reviewed above, has been extended to the case of guided-wave structures that can have lateral dimensions smaller than the conventional diffraction limit $\lambda / 2$. [26], [27] When the structure shown in Fig. 3(A) is used as a parallel-plate waveguide with the DPS-DNG pair, the dispersion relation for guided mode with longitudinal waveguide wavenumber $\beta$ has been shown to be

$$
\begin{aligned}
\frac{\tan \left(k_{t \mathrm{DPS}}^{\mathrm{TE}} d_{\mathrm{DPS}}\right)}{\tan \left(k_{t \mathrm{DNG}}^{\mathrm{TE}} d_{\mathrm{DNG}}\right)} & =-\frac{\mu_{\mathrm{DNG}} k_{t \mathrm{DPS}}^{\mathrm{TE}}}{\mu_{\mathrm{DPS}} k_{t \mathrm{DNG}}^{\mathrm{TE}}} \quad \text { TE modes } \\
\frac{\cot \left(k_{t \mathrm{DPS}}^{\mathrm{TM}} d_{\mathrm{DPS}}\right)}{\cot \left(k_{t \mathrm{DPS}}^{\mathrm{TM}} d_{\mathrm{DPS}}\right)} & =-\frac{\varepsilon_{\mathrm{DNG}} k_{t \mathrm{DPS}}^{\mathrm{TM}}}{\varepsilon_{\mathrm{DPS}} k_{t \mathrm{DNG}}^{\mathrm{TM}}} \quad \mathrm{TM} \text { modes }
\end{aligned}
$$

where $k_{t}^{\mathrm{TE}} \equiv \sqrt{k^{2}-\beta_{\mathrm{TE}}^{2}}$ for the TE mode and a corresponding expression for the TM mode [26]. For DPS and DNG slabs, $k^{2}=\omega^{2} \varepsilon_{\mathrm{DPS}} \mu_{\mathrm{DPS}}>0$ and $k^{2}=\omega^{2} \varepsilon_{\mathrm{DNG}} \mu_{\mathrm{DNG}}>0$, respectively, and the transverse wavenumber $k_{t \mathrm{DPS}}$ and $k_{t \mathrm{DNG}}$ may be real or imaginary, depending on the value of $\beta$. In standard metallic waveguides filled with DPS materials, it is known that guided modes cannot be supported when the lateral dimensions fall below a certain limit, i.e., below the cutoff thickness. In other words, in a closed waveguide with a metallic wall, reducing the cross-sectional size of the guide results in cutting off the modes one after the other until the dominant mode is also cut off. However, in the DPS-DNG or ENG-MNG waveguides, it may be possible to overcome this limitation and to devise a waveguide capable of supporting guided modes without any cutoff thickness [26], [27]. By properly choosing the material parameters and thicknesses of the DPS and DNG layers in the waveguide, one can obtain various interesting features for such a waveguide. Some of these properties for the case of parallel-plate waveguides are summarized below.

Fig. 4(A) presents a sample of the dispersion diagram for the TE mode in the DPS-DNG parallel-plate waveguide, showing the relationship among the normalized total thickness $d_{\mathrm{DPS}}+d_{\mathrm{DNG}}, d_{\mathrm{DPS}}$, and $\beta_{\mathrm{TE}}$. For comparison, the corresponding TE-mode dispersion diagram for the DPS-DPS parallel-plate waveguide is shown in Fig. 4(B).

Referring to Fig. 3(A) as the geometry of the waveguide and Fig. 4 for its dispersion diagram, let us first consider the case where the DPS and DNG layers are very thin, i.e.,
$\left|k_{\mathrm{DPS}}\right| d_{\mathrm{DPS}} \ll 1$ and $\left|k_{\mathrm{DNG}}\right| d_{\mathrm{DNG}} \ll 1$. In this case, the dispersion relations in (7) may be approximated, respectively, as

$$
\begin{gathered}
\gamma \simeq-\frac{\mu_{\mathrm{DNG}}}{\mu_{\mathrm{DPS}}}=\frac{\left|\mu_{\mathrm{DNG}}\right|}{\mu_{\mathrm{DPS}}} \quad \text { TE case } \\
\beta_{\mathrm{TM}} \simeq \pm \omega \sqrt{\frac{\mu_{\mathrm{DPS}}+\mu_{\mathrm{DNG}}}{\frac{\gamma}{\varepsilon_{\mathrm{DPS}}}+\frac{1}{\varepsilon_{\mathrm{DNG}}}}} \quad \text { TM case }
\end{gathered}
$$

where $\gamma$ is shorthand for $d_{\mathrm{DPS}} / d_{\mathrm{DNG}}$ and obviously should always be a positive quantity. If instead of a pair of DPS-DNG layers, this thin waveguide were filled with a pair of DPS-DPS layers (and similarly with a pair of ENG-ENG, DPS-ENG, MNG-MNG, DNG-MNG, or DNG-DNG layers), the first equation in (8) would not be satisfied because, for these pairs, the right-hand side of that equation would not be positive and, thus, no TE mode could propagate in such a thin DPS-DPS waveguide, as expected. This can be seen from Fig. 4(B) around the region of the diagram where thickness of one of the layers (e.g., the first DPS layer) is taken to be very small, and we note that, in this region, the total thickness of the guide cannot be lower than a certain limit. This indeed would represent the diffraction limit mentioned above for standard waveguides. However, if the thin waveguide is filled with a pair of DPS-DNG slabs (or a pair of DNG-ENG, DPS-MNG, or ENG-MNG slabs), the right-hand side of the first equation in (8) would be positive and, thus, that equation may be approximately satisfied for a certain value of $\gamma$. In this case, the exact expression for $\beta_{\mathrm{TE}}$ should be obtained by solving the TE dispersion relation in (7). As we can see from that equation and also from Fig. 4(A), the region where both $\left|k_{\mathrm{DPS}}\right| d_{\mathrm{DPS}} \ll 1$ and $\left|k_{\mathrm{DNG}}\right| d_{\mathrm{DNG}} \ll 1$ is allowed and, hence, the total thickness $d_{\mathrm{DPS}}+d_{\mathrm{DNG}}$ can be very small, contrary to the case of the DPS-DPS waveguide. In such a limit and as long as the TE dispersion relation in (7) is satisfied, one (and only one) propagating TE mode may exist in principle, as Fig. 4(A) shows, no matter how thin these layers are. This means a parallel-plate waveguide filled with DPS-DNG layers does not have a cutoff thickness for the TE modes; thus, it can support a TE guided mode, even though the lateral dimension can be well below the diffraction limit.

As for the TM mode, the second equation in (8) provides the approximate value for $\beta_{\mathrm{TM}}$ of the dominant TM mode when $\beta_{\mathrm{TM}}$ is a real quantity for a given set of $\gamma$ and material parameters. For a DPS-DPS or DNG-DNG thin waveguide, this TM mode exists for any ratio $\gamma$, and its $\beta_{\mathrm{TM}}$ lies between the wavenumbers of the two layers. The allowable ranges of variation of $\beta_{\mathrm{TM}}$ in (8) in terms of $\gamma$ have been discussed in detail in [26] and [27]. It has been shown that, for the DPS-DNG parallel-plate waveguide, the range of variation of $\beta_{\mathrm{TM}}$ indeed differs from the ones in the standard DPS-DPS waveguides in that $\beta_{\mathrm{TM}}$ may attain values only outside the interval between $\left|k_{\mathrm{DPS}}\right|$ and $\left|k_{\mathrm{DNG}}\right|$ (effectively "complementary" to the standard DPS-DPS case where $\beta_{\mathrm{TM}}$ is in this interval). The fact that thin waveguides loaded with complimentary pairs of MTMs (e.g., DPS-DNG or ENG-MNG) may support nonlimited $\beta_{\mathrm{TM}}$, may offer interesting possibilities in the design of very thin resonant cavities and very thin waveguides having guided modes with high $\beta$. With $\beta \gg 1$, the waveguide wavelength $\lambda_{g}=2 \pi / \beta$ will be very small, which 

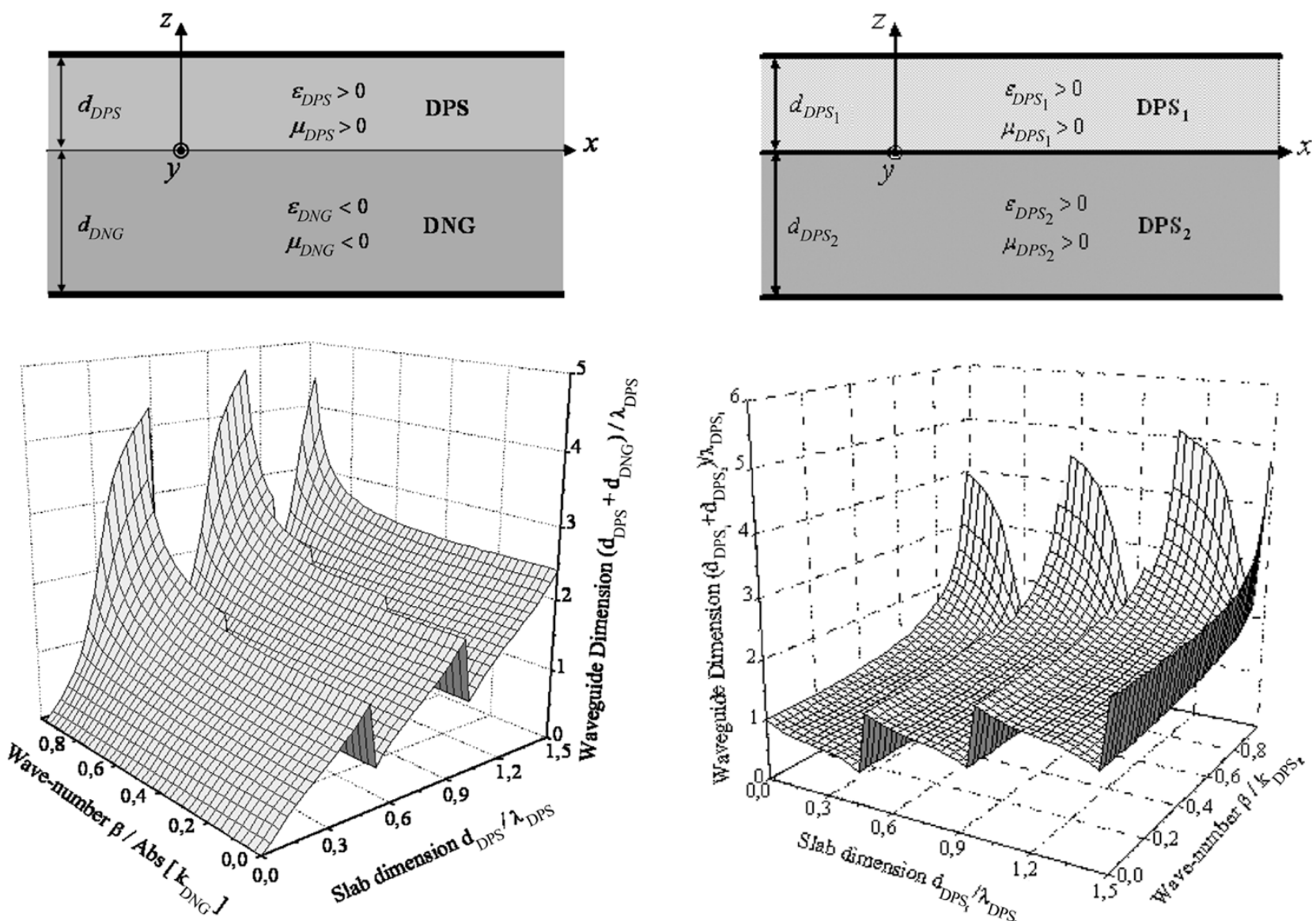

(A)

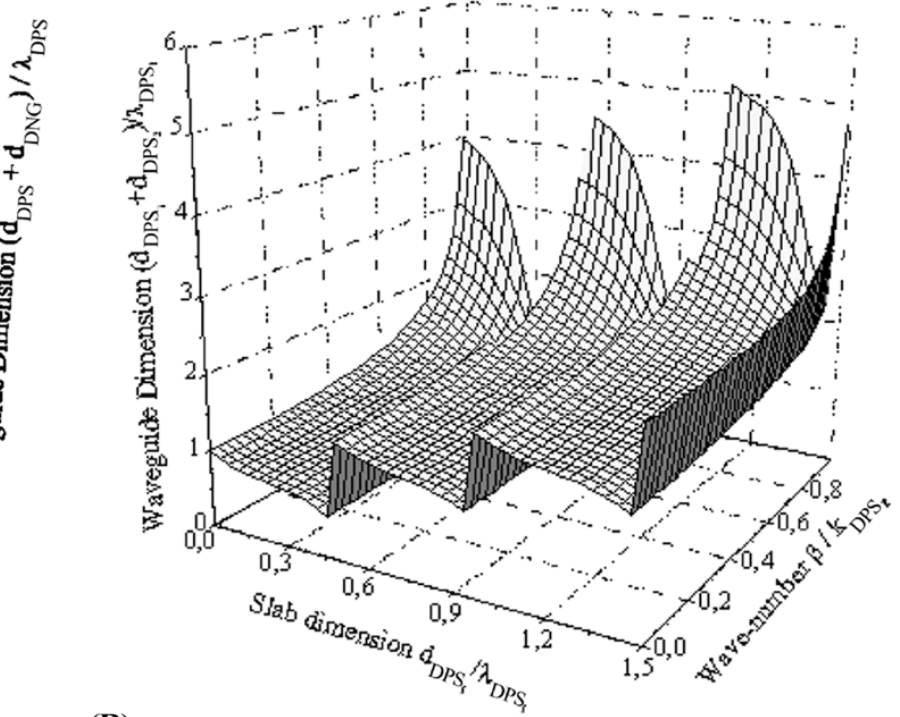

Fig. 4. Dispersion plots for: (A) the DPS-DNG parallel-plate waveguide and (B) the DPS-DPS parallel-plate waveguide for comparison. Minimum total thickness $\left(d_{\mathrm{DPS}}+d_{\mathrm{DNG}}\right) / \lambda_{\mathrm{DPS}}$ for the waveguide is shown in terms of $\beta /\left|k_{\mathrm{DNG}}\right|$ and $d_{\mathrm{DPS}} / \lambda_{\mathrm{DPS}}$. Here, we have $\varepsilon_{1}=3 \varepsilon_{o}, \mu_{1}=3 \mu_{o}, \varepsilon_{2}=\mp 3 \varepsilon_{o}$, and $\mu_{2}=\mp \mu_{o}$ where the upper signs are for (A) with DPS-DNG and the lower signs are for (B) with DPS-DPS. From [76].

may give rise to compact resonators and filters. A similar observation regarding the possibility of $\beta_{\mathrm{TM}}$ being very large has also been made by Nefedov and Tretyakov [61]. Other salient features of DPS-DNG waveguides such as unusual properties in mode excitation, backward flow of power in the DPS-DNG waveguides, and the effect of geometric discontinuity in the DPS-DNG slabs in such waveguides have been studied and reported in the literature [27], [76]. Moreover, some of the unconventional characteristics of such DPS-DNG waveguides and cavities can be explained and justified using the distributed-circuit-element approach with appropriate choice of elements [77]. This "circuit-element" approach may also be applied to ENG-MNG, or DPS-SNG waveguides [77]. It is worth noting that there are other techniques to construct subwavelength waveguides by embedding split-ring resonators in a waveguide below cutoff [117], [118]. These split-ring resonators can be considered as lumped elements within such subwavelength waveguides, whereas the structures considered above are subwavelength due to the resonant pairing of the DPS and DNG layers.

The case of cylindrical waveguide with a metallic wall filled with coaxial layers of DPS-DNG or ENG-MNG materials have also been studied by Alù and Engheta [73], and analogous results have been obtained.

Guided-wave structures involving SNG materials have also been analyzed in detail by Alù and Engheta [26], [27], and similarities and differences between the ENG-MNG and DPS-DNG parallel-plate waveguides have been discussed and reported [26]. For thin waveguides, these two sets of waveguide have many similarities. However, as the parallel-plate waveguides gets thicker, the differences between these two classes of waveguide become evident. For example, we have shown that it is possible to have a mono-modal thick parallel-plate waveguide filled with a pair of ENG and MNG layers, whereas the same size DPS-DNG waveguide may support more than one mode. Other characteristics of the SNG waveguides can be found in [26] and [27].

The case of surface-wave propagation along the open ungrounded DNG slab waveguides has also been studied [27]. The geometry of such a DNG waveguide is shown in Fig. 5(A).

The dispersion relation is given as

$$
\begin{aligned}
& \frac{\mu_{\mathrm{DNG}}}{\sqrt{k_{\mathrm{DNG}}^{2}-\beta_{\mathrm{even}}^{2}}} \cot \left(\sqrt{k_{\mathrm{DNG}}^{2}-\beta_{\mathrm{even}}^{2}} d\right)=\frac{\mu_{o}}{\sqrt{\beta_{\mathrm{even}}^{2}-k_{o}^{2}}} \\
& \frac{\mu_{\mathrm{DNG}}}{\sqrt{k_{\mathrm{DNG}}^{2}-\beta_{\mathrm{odd}}^{2}}} \tan \left(\sqrt{k_{\mathrm{DNG}}^{2}-\beta_{\mathrm{odd}}^{2}} d\right)=-\frac{\mu_{o}}{\sqrt{\beta_{\mathrm{odd}}^{2}-k_{o}^{2}}}
\end{aligned}
$$

where $k_{o}^{2} \equiv \omega^{2} \mu_{o} \varepsilon_{o}$ and $d$ is half of the slab thickness. Fig. 5(B) shows the dispersion diagrams for the odd TE mode in the open DNG slab waveguide (solid line) and the corresponding DPS slab waveguide (dashed line), where $\Delta k^{2} \equiv k_{\mathrm{DNG}}^{2}-k_{o}^{2}$. One 


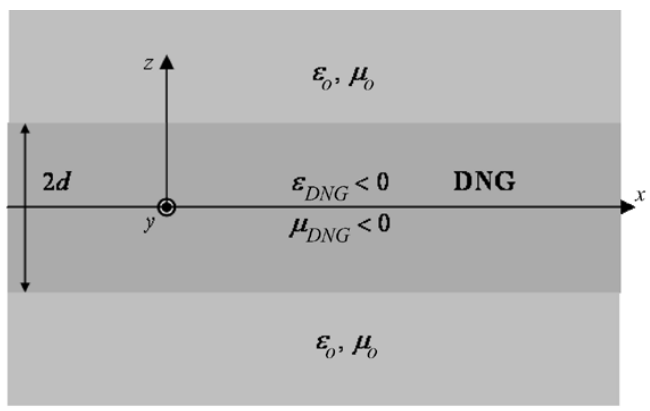

(A)

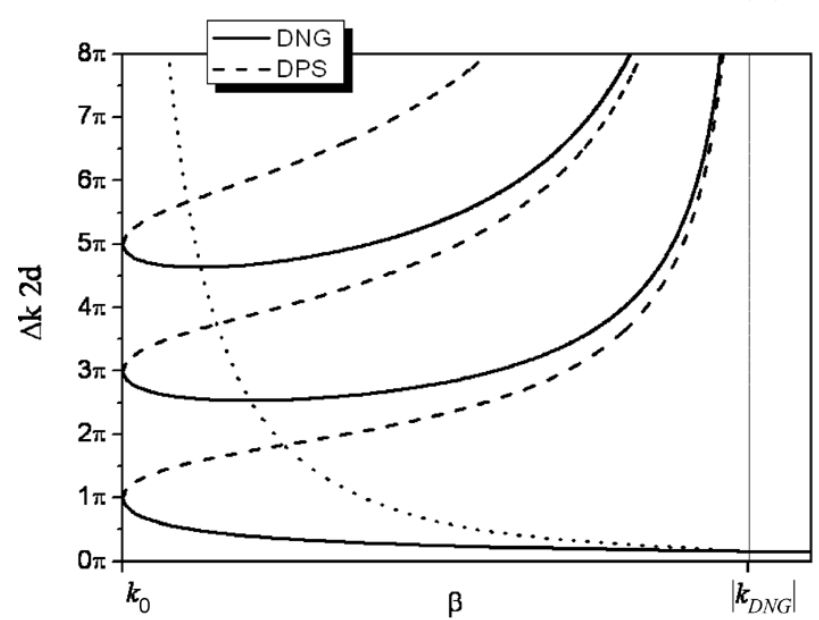

(B)

Fig. 5. (A) Open ungrounded DNG slab waveguide. (B) Its dispersion diagram (solid line) for the odd TE mode with $\mu_{\mathrm{DNG}}=-4 \mu_{0}$ and $\varepsilon_{\mathrm{DNG}}=-2 \varepsilon_{0}$. The dispersion diagram for the corresponding open DPS slab waveguide with $\mu_{\mathrm{DPS}}=4 \mu_{o}$ and $\varepsilon_{\mathrm{DPS}}=2 \varepsilon_{o}$ is shown with the dashed line.

striking difference between the two dispersion diagrams is the presence of a TE odd mode in the DNG slab as the expression $\Delta k 2 d$ tends to small values below $\pi$. We also note that, for this solution of the dispersion relation (i.e., the lowest solid line in Fig. 5(B)), the value of $\beta_{\text {odd }}$ increases as $\Delta k 2 d \rightarrow 0$. In fact, $\beta_{\text {odd }}$ can become even greater than $k_{\mathrm{DNG}}$.

In order to highlight the importance of this solution, let us first consider the case of a standard DPS slab waveguide in which propagating guided modes are possible only when $k_{o}<\beta<$ $k_{\text {DPS. }}$. In particular, the first even mode has no cut off, i.e., even when $d \rightarrow 0$, a solution for $\beta_{\text {even }}$ still exists and is $\beta_{\text {even }} \rightarrow$ $k_{0}+0$. However, this DPS behavior also implies that the lateral distribution of the field of such a guided mode is widespread in the region surrounding the slab, and essentially the mode is only weakly guided. Therefore, when the guiding structure with the DPS material becomes very thin, the effective cross section of the guided mode becomes very large, i.e., in the limit of zero slab thickness, the guided mode is simply a uniform plane wave. Consequently, if we consider reducing the slab thickness, the guided mode will travel with a transverse section much larger than the slab lateral dimension. This issue is indeed another manifestation of the diffraction limitation, which does not traditionally allow the signal transport in a guided structure thinner than a given dimension determined by the wavelength of operation.

For the DNG slab waveguide, the situation is quite different. In this case, since the first odd mode has no cutoff thickness, the fields are more concentrated and confined near the slab surface. This is an important advantage of such DNG open-slab waveguides. It implies that the guided surface wave can be present even for a sub-wavelength guided-wave structure with lateral dimension below the diffraction limit and that it will be tightly confined to this structure since $\beta_{\text {odd }} \gg k_{0}$. Several other details about the dispersion properties of the open ungrounded DNG slab waveguides can be found in [27], among those one can mention the fact that such a waveguide supports guided modes in which the portion of the power flowing in the surrounding vacuum is antiparallel with respect to the portion flowing inside the DNG slab. This feature can offer an interesting possibility for the "backward" coupling between such DNG open waveguides and standard DPS open waveguide placed in their proximity [27], [78]. This will be briefly reviewed in Section VI. It is also important to note that the properties of the grounded DNG slab has been studied by Baccarelli et al. with an emphasis toward conditions for the absence of surface waves in such grounded structures with potential application to printed antenna systems [79].

The possibility of having guided modes with $\beta_{\text {odd }} \gg k_{o}$ can provide a possible solution for the transport of RF and optical energy in structures with small lateral dimensions, below the diffraction limit with possible applications to miniaturization of optical interconnects and nanophotonics. Ideally, in the lossless case, there is, in principle, no limitation on the compactness of such waveguides and the confinement of the guided mode. However, in practice, loss is present and may limit the performance and, thus, should be taken into consideration.

Similar characteristics have also been obtained for the thin open cylindrical waveguides formed by a DNG or a SNG cylinder or coaxial layers of DNG-DPS or DPS-SNG materials [80].

\section{BACKWARD COUPLERS USING DNG SLAB WAVEGUIDES}

As mentioned above, owing to the peculiar behavior of guided modes in open DNG slab waveguides, they can exhibit backward coupling properties when they are in proximity of open DPS slab waveguides, i.e., if one of the two waveguides is excited to carry power in one direction, the second waveguide, through the coupling, might "redirect" back some of this power in the opposite direction. We should note that an analogous phenomenon for the planar circuits has been observed and studied in the negative-index transmission-line couplers by Islam et al. [45] and Caloz and Itoh [42].

The geometry of the problem is shown in Fig. 6, in which the two open-slab waveguides are separated and surrounded by a simple medium (e.g., free space). In this figure, a simple physical description of the backward coupling of the power flows in the two slabs is also given in terms of the phase and Poynting vectors. Using a rigorous modal analysis of the structure, the following dispersion relation for the supported TE modes can be obtained:

$$
\operatorname{Disp}_{1} \operatorname{Disp}_{2}=c_{1} c_{2}
$$

where $\operatorname{Disp}_{i}=0$ (with $i=1,2$ ) with

$$
\begin{aligned}
\operatorname{Disp}_{i}= & \left(\sqrt{k_{i}^{2}-\beta^{2}} \cot \left(\sqrt{k_{i}^{2}-\beta^{2}} \frac{d_{i}}{2}\right)+\mu_{i} \sqrt{\beta^{2}-k_{0}^{2}}\right) \\
& \cdot\left(\sqrt{k_{i}^{2}-\beta^{2}} \tan \left(\sqrt{k_{i}^{2}-\beta^{2}} \frac{d_{i}}{2}\right)-\mu_{i} \sqrt{\beta^{2}-k_{0}^{2}}\right)
\end{aligned}
$$

is the modal dispersion relation of each slab alone (i.e., without coupling), represented by the product of the dispersion rela- 


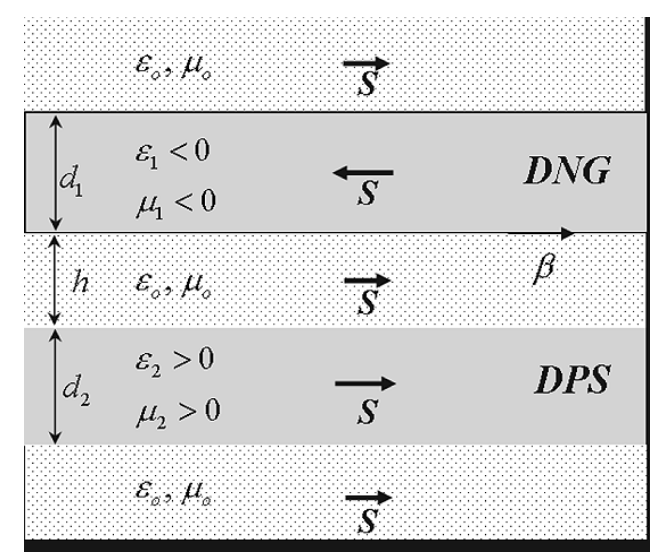

Fig. 6. Geometry of the backward coupler with the DNG and DPS slab waveguides with a sketch of the power flows for a given supported mode. From [78].

tions of even and odd TE modes in the isolated slab, $\beta$ is the waveguide wavenumber, $k_{i} \equiv \omega \sqrt{\mu_{i}} \sqrt{\varepsilon_{i}}$ (with $i=1,2$ ), and $k_{o} \equiv \omega \sqrt{\mu_{o} \varepsilon_{o}}$. The coefficients on the right-hand side of (10) take into account the coupling effects and are given by

$$
\begin{aligned}
c_{i}=\frac{1}{2} e^{-h \sqrt{\beta^{2}-k_{0}^{2}}}\left[\beta^{2}\left(\mu_{i}^{2}-\mu_{0}^{2}\right)\right. & \left.+\mu_{0}^{2} k_{i}^{2}-\mu_{i}^{2} k_{0}^{2}\right] \\
\times & \sin \left(\sqrt{k_{i}^{2}-\beta^{2}} d_{i}\right) .
\end{aligned}
$$

The TM mode dispersion relation may be straightforwardly obtained using the duality principle. When the two waveguides are far apart (i.e., $h$ is sufficiently large), the coupling term on the right-hand side of the dispersion relation vanish and, as expected, (10) reduces into the dispersion relations for the two "decoupled" open waveguides. The modes in each waveguide are unperturbed and, thus, there is no coupling present. When $h$ is reduced, however, the modes supported by each one of the two waveguides have field distributions that extend into the region occupied by the other waveguide. Thus, a new set of modes should be found to satisfy the exact dispersion relation with a field distribution obtainable by solving the boundary value problem. The properties of these modes can be obtained by using perturbation techniques or the exact formulation. Some of the details can be found in [27]. Here, we briefly describe an interesting distinction between a DPS-DNG waveguide coupler and a corresponding standard DPS-DPS waveguide coupler. In a standard waveguide directional coupler, when we fix the geometry of the two waveguides separately and, therefore, fix the values of $\beta_{1}^{\text {no coupling }}$ and $\beta_{2}^{\text {no coupling }}$ without coupling, the exact solutions for the wavenumbers $\beta_{1}^{\text {coupling }}$ and $\beta_{2}^{\text {coupling }}$ move farther from each other as $h$ is reduced. Moreover, their interference spatial period consequently decreases. When instead we consider a DPS-DNG backward coupler, the two solutions $\beta_{1}^{\text {coupling }}$ and $\beta_{2}^{\text {coupling }}$ move closer as $h$ is reduced, thus increasing the spatial period of their coupling. One can then get to a point at which the two supported modes have the same wavenumber, i.e., $\beta_{1}^{\text {coupling }}=\beta_{2}^{\text {coupling; }}$; and the interference is no longer present (i.e., its period is infinite). By decreasing the distance $h$ further and, hence, increasing the coupling coefficient, an exponential variation for the power exchange (rather than a sinusoidal variation) results. Consequently, the power is "redirected" back into the other waveguide continuously and exponentially with a factor that increases as $h$ decreases. In the exact approach, this behavior is due to the fact that the two modes share the same real part, but start to have two oppositely valued imaginary parts. The backward coupler with a strong coupling, therefore, acts similarly to a periodically corrugated waveguide (grating reflector [122]) in its stopband, but with the unusual feature that the "reflected" power is effectively flowing in a separate channel and is isolated from the "incident" one. This implies that the incident and reflected power flows are spatially localized in the two different waveguides.

\section{Sub-WaVelength Focusing With a Flat Lens oR A CONCAVE LENS MADE OF DNG MATERIALS}

Another one of the interesting potential applications of DNG media, which was first theoretically suggested by Pendry [64], is the idea of a "perfect lens" or focusing beyond the diffraction limit. In his analysis of the image formation process in a flat slab of lossless DNG material, Pendry showed that, in addition to the faithful reconstruction of all the propagating spatial Fourier components, the evanescent spatial Fourier components can also ideally be reconstructed. The evanescent wave reconstruction is due to the presence of a "growing exponential effect" in the DNG slab, leading to the formation of an image with a resolution higher than the conventional limit. His idea has motivated much interest in studying wave interaction with DNG media. Various theoretical and experimental works by several groups have explored this possibility; they have shown the possibility and limitations of sub-wavelength focusing using a slab of DNG or negative-index MTMs [81], [82]. The sub-wavelength focusing in the planar 2-D structures made of negative-index transmission lines has also been investigated [46]. The presence of the growing exponential in the DNG slab has also been explained and justified using the equivalent distributed circuit elements in a transmission-line model [83]. It has also been shown that "growing evanescent envelopes" for the field distributions can be achieved in a suitably designed, periodically layered stacks of frequency selective surfaces (FSSs) [84].

In Fig. 7(A), we present an FDTD simulation of the focusing effects for a planar slab of DNG materials with $n_{\text {real }}\left(\omega_{0}\right) \approx-1$. A diverging $\mathrm{CW}$-modulated Gaussian beam is assumed to be normally incident on such a planar DNG slab in order to determine whether it can focus such a diverging Gaussian beam or not. The focal plane (or waist) of the beam was taken to be in front of the slab. Since it was expected that the DNG slab would have an NIR and would focus the beam, i.e., it would bend the wave vectors of a diverging beam back toward the beam axis, a strongly divergent beam was considered in this simulation. A diffraction limited beam, whose waist was approximately $\lambda_{o} / 2$, was used. The location of the waist was set at $2 \lambda_{o}$ away from the DNG interface so that there would be sufficient distance for the beam to diverge before it hit the interface. The DNG slab also had a depth of $2 \lambda_{o}$. Therefore, if the DNG slab refocuses the beam, the waist of the beam at the back face of the DNG slab should be approximately the same as its initial value (the Drude medium has some small losses). A snapshot of the FDTD predicted electric-field intensity distribution is shown in Fig. 7(A). This result clearly shows that the planar DNG medium turns the 
(A)

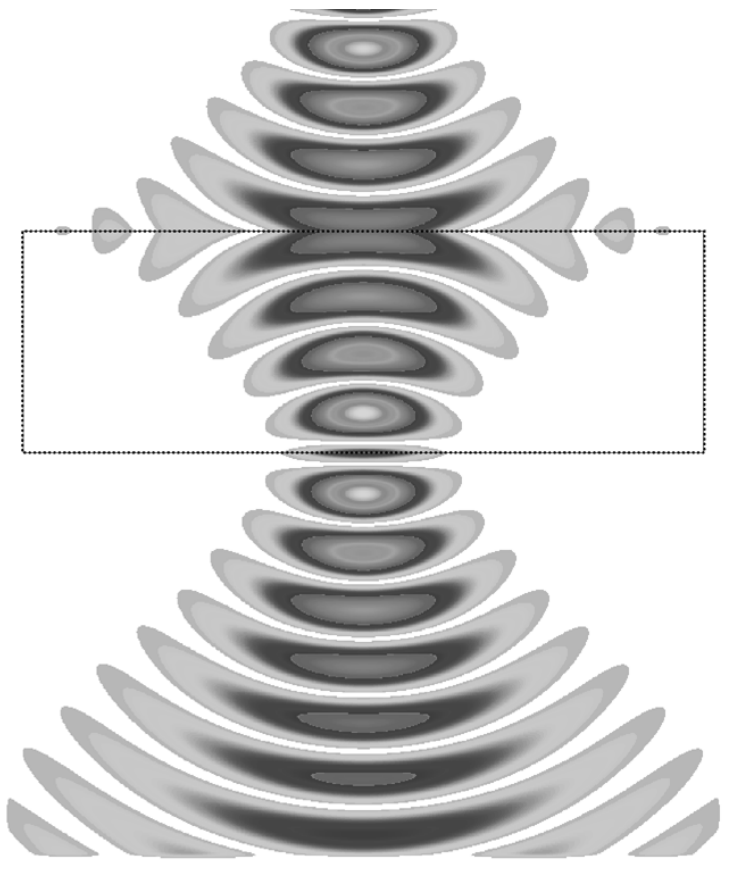

(B)

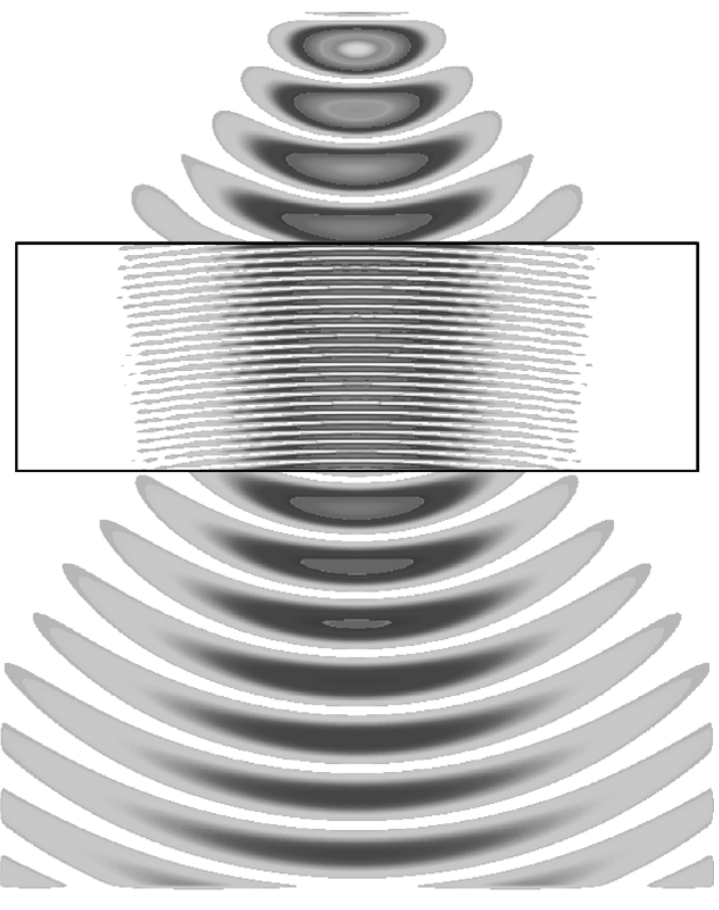

Fig. 7. (A) FDTD predicted electric-field intensity distributions illustrate the focusing of the Gaussian beam as it propagates in the $n_{\text {real }}\left(\omega_{0}\right) \approx-1$ DNG slab. Focusing at the back surface is observed. (B) Similar FDTD simulation for a DNG slab having $n_{\text {real }}\left(\omega_{0}\right) \approx-6$. Channeling of the beam in the DNG slab is observed; the wings of the beam are seen to feed the center of the beam.

diverging wave vectors toward the beam axis and, hence, acts as a lens to focus the beam. Since all angles of refraction are the negative of their angles of incidence for the $n_{\text {real }}\left(\omega_{0}\right) \approx-1$ slab, the initial beam distribution is essentially recovered at the back face of the slab, i.e., as designed, the focal plane of the beam in the DNG medium is located at the back face of the DNG slab. From the electric-field intensity obtained from the FDTD simulation, we note that the peak intensity is approximately $18 \%$ lower than its value at the original waist of the Gaussian beam.
This variance stems from the presence of additional wave processes, such as surface-wave generation, and from dispersion and loss in the actual Drude model used to define the DNG slab in the FDTD simulation.

We note that if, as shown by Ziolkowski and Heyman in [12], a point source is at a distance $d / 2$ from the front face of an $n_{\text {real }}\left(\omega_{0}\right) \approx-1$ planar slab of thickness $d$, then the first focus of the source is found in the center of the slab a distance $d / 2$ from the front face and the second focus is located beyond the slab at a distance $d / 2$ from the back face. The slab effectively translates the source to the exterior focus position. If the reconstruction by the growing evanescent fields in the slab is to achieve sub-wavelength focusing at the image point and if the slab has even a small amount of loss, the slab will have to be thin. The source and its image will then have to be very near, respectively, to the front and back faces of the slab. The configuration will then essentially become a near-field one, and the "perfect lens" situation is thus lost to only near-field configurations when real media with losses are involved. Nonetheless, since there are numerous near-field imaging situations, such as breast tumor sensing, the slab translator may still provide interesting imaging possibilities. We note that if the source is moved further away from the front face of the slab, the foci inside and outside the slab will move closer to the back face of the slab. Consequently, even a normal lens configuration in which the source is far from the lens, the "perfect lens" only becomes useful in the near field of the output face of the system.

The corresponding results for the Gaussian beam interacting with the matched DNG slab with $n_{\text {real }}\left(\omega_{0}\right) \approx-6$, shown in Fig. 7(B), reveals related, but different results. In contrast to the $n_{\text {real }}\left(\omega_{0}\right) \approx-1$ case, when the beam interacts with the matched DNG slab with $n_{\text {real }}\left(\omega_{0}\right) \approx-6$, there is little focusing observed. The negative angles of refraction dictated by Snell's Law are shallower for this higher magnitude of the refractive index, i.e., $\theta_{\text {trans }} \approx-\sin ^{-1}\left[\sin \left(\theta_{\text {inc }}\right) / 6\right]$. Rather than a strong focusing, the medium channels power from the wings of the beam toward its axis, hence, maintaining its amplitude as it propagates into the DNG medium. The width of the beam at the back face is only slightly narrower, yielding only a slightly higher peak value there in comparison to its values at the front face. The strong axial compression of the beam caused by the (factor of six) decrease in the wavelength in the $n_{\text {real }}\left(\omega_{0}\right) \approx-6$ slab also occurs. We note that, in these DNG cases, the beam appears to diverge significantly once it leaves the DNG slab. The properties of the DNG medium hold the beam together as it propagates through the slab. Once it leaves the DNG slab, the beam must begin diverging, i.e., if the DNG slab focuses the beam as it enters, the same physics will cause the beam to diverge as it exits. Moreover, there will be no focusing of the power from the wings to maintain the center portion of the beam. The rate of divergence of the exiting beam will be determined by its original value and the properties and size of the DNG medium. We also point out that a beam focused into a DNG slab will generate a diverging beam within the slab and a converging beam upon exit from the slab. This behavior has also been confirmed with the FDTD simulator.

One potential application for these results is clearly the use of a matched flat DNG slab with an index of refraction $n_{\text {real }}\left(\omega_{0}\right) \approx$ -1 as a lens/translator, as originally suggested by Pendry [64]. 
This can have various applications, for instance, in a variety of near-field microwave or optical systems. Another potential application is to channel the field into a particular location, e.g., to use a large negative index DNG slab (e.g., $n_{\text {real }}\left(\omega_{0}\right) \approx-6$ ) as a superstrate (over-layer) on a detector so that the beam energy would be channeled efficiently onto the detector's face. Most superstrates, being simple dielectrics, defocus the beam. Often one includes a curved DPS lens over the detector face to achieve the focusing effect. The flatness of the DNG slab has further advantages in packaging the detectors into an array or a system. Yet another potential application is to combine the negative index properties of the DNG slab with its negative refraction features to realize a low-loss phase compensator, as discussed earlier.

It should be mentioned that a planar DNG slab is unable to focus a collimated beam (i.e., flat beam) or a plane wave since the negative angle of refraction can occur only if there is oblique incidence. This is the reason why only FDTD simulations for expanding Gaussian beams have been shown up to this point. In order to focus a flat Gaussian beam (one with nearly an infinite radius of curvature), one must resort to a curved lens. However, in contrast to focusing (diverging), a plane wave with a convex (concave) lens composed of a DPS medium, here one must consider focusing (diverging) a plane wave with a concave (convex) lens composed of a DNG medium. Such a plano-concave DNG lens with $n_{\text {real }}\left(\omega_{0}\right) \approx-1$ is shown in Fig. $8(\mathrm{~A})$ for the FDTD simulation. It was formed by removing a parabolic section from the backside of a slab that was $1.5 \lambda_{0}$ deep and $6.0 \lambda_{0}$ wide. The focal length was chosen to be $\lambda_{0}$, and the location of the focus was chosen to be at the center of the back face of the slab. The full width of the removed parabolic section at the back face was $4 \lambda_{0}$. A Gaussian beam with a waist of $2 \lambda_{0}$ was launched $2 \lambda_{0}$ distance away from the planar side of this lens and was normally incident on it.

It is known that a DPS plano-convex lens of index $n_{\text {DPS }}$ with a similar radius of curvature $R=2 \lambda_{0}$ [the dark gray region in Fig. 8(A)] would have a focus located a distance $f_{\mathrm{DPS}}=$ $R /\left(n_{\mathrm{DPS}}-1\right)=2 \lambda_{0} /\left(n_{\mathrm{DPS}}-1\right)$ from its back face. Thus, to have the focal point within the very near field, as it is in the DNG case, the index of refraction would have to be very large. In fact, to have it located at the back face would require $n_{\text {DPS }} \rightarrow \infty$. This would also mean that very little of the incident beam would be transmitted through such a high index lens because the magnitude of the reflection coefficient would approach one. In contrast, the DNG lens achieves a greater bending of the incident waves with only moderate absolute values of the refractive index and is impedance matched to the incident medium. Moreover, since the incident beam waist occurs at the lens, the expected waist of the focused beam would be $w_{\text {focus }} \approx$ $\left(\lambda_{0} f_{\mathrm{DPS}}\right) /\left(\pi w_{0}\right)=\lambda_{0} /\left[\pi\left(n_{\mathrm{DPS}}-1\right)\right]$ [123]. Hence, for a normal glass lens $n_{\mathrm{DPS}} \approx 1.5$, the transverse waist at the focus would be $w_{\text {focus }} \approx \lambda_{0} / 1.57$ and the corresponding intensity half-max waist would be $0.589 w_{\text {focus }}$. The longitudinal size of the focus is the depth of focus, which, for the normal glass lens would be $2\left(\pi w_{\text {focus }}^{2} / \lambda_{0}\right)$. Again, to achieve a focus that is significantly sub-wavelength using a DPS lens, a very large index value would be required and would lead to similar disadvantages in comparison to the DNG lens. However, for the DNG plano-concave lens, one obtains more favorable results.

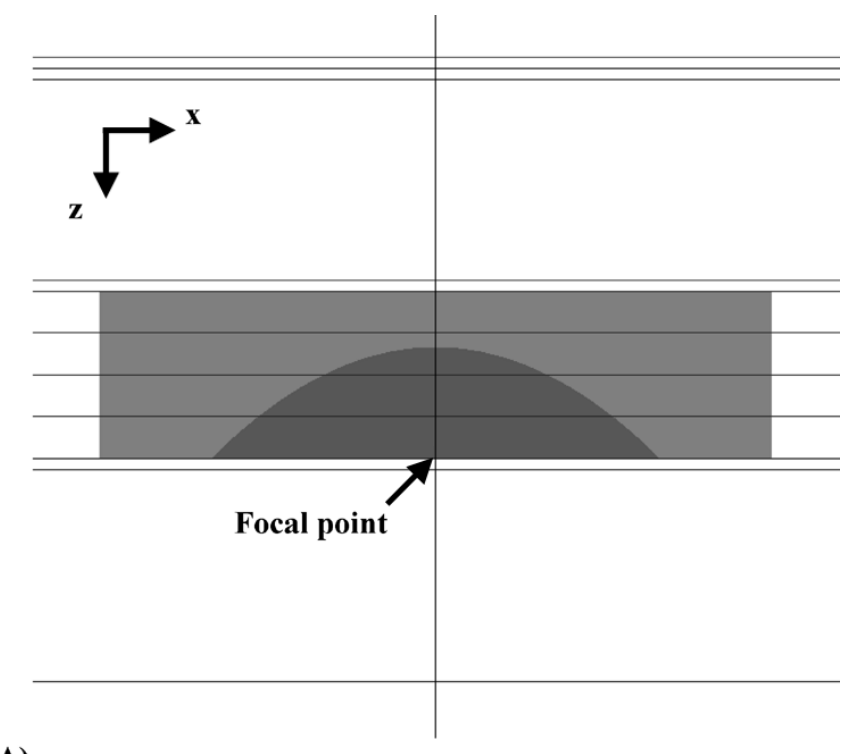

(A)

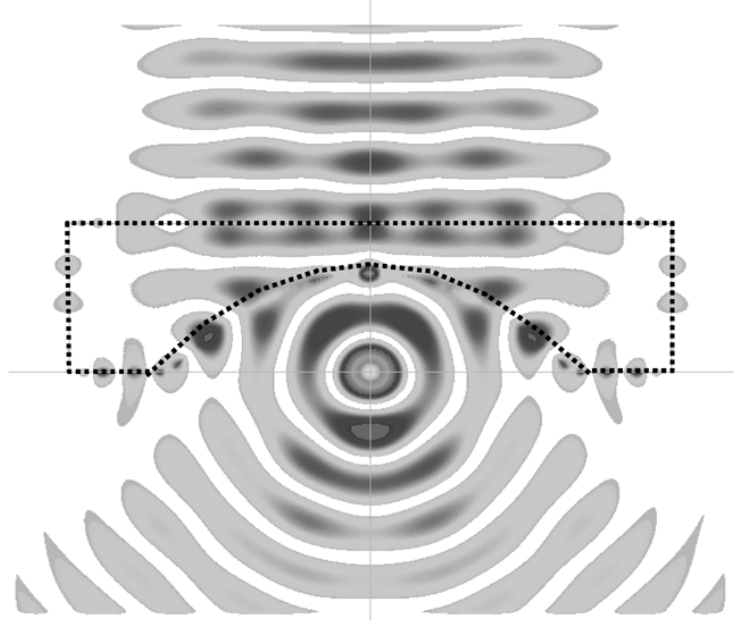

(B)

Fig. 8. (A) Plano-concave DNG lens geometry for the FDTD simulation. The launching plane and beam axis are shown by the black lines. The electric-field sampling points were located at the intersections of the beam axis and horizontal lines. The location of the DNG lens region is shown in light gray. The dark gray region is air, as is the entire region surrounding the lens. (B) Snapshot of the FDTD-predicted electric-field intensity distributions for the DNG concave lens. The peak of the intensity occurs at the predicted focal point. This sub-wavelength focal region is significantly smaller than would be expected from the corresponding traditional DPS lens.

Fig. 8(B) shows a snapshot of the FDTD-predicted electric-field intensity distribution when the intensity is peaked at the focal point. The radius of the focus along the beam axis (half intensity radius) is measured to be approximately $\lambda_{0} / 5$ and along the transverse direction it is approximately $\lambda_{0} / 6$. This sub-wavelength focal region is significantly smaller than would be expected from the corresponding traditional DPS lens. Moreover, even though the focal point is in the extreme near field of the lens, the focal region is nearly symmetrical and has a resolution that is much smaller than a wavelength. Such a sub-wavelength source has a variety of desirable features that may have applications for high-resolution imaging in near-field scanning optical microscopy (NSOM) systems. In particular, the field intensity has been concentrated into a sub-wavelength region without a 


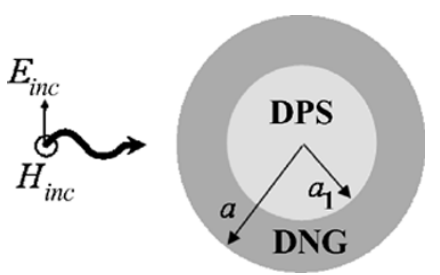

Fig. 9. Spherical scatterer composed of two concentric shells of DPS and DNG isotropic materials.

guiding structure. It could thus act as a much smaller aperture NSOM source than is available with a typical tapered optical fiber probe and without the associated aperture effects.

\section{SUB-WAVELENGTH SPHERICAL AND CYLINDRICAL RESONANT StruCtures CONTAINING DNG METAMATERIALS}

Earlier we reviewed the possibility of designing a sub-wavelength 1-D cavity resonator by pairing DPS and DNG layers. This resonance phenomenon can be intuitively explained by noting that if the DPS layer acts as an equivalent reactive impedance, the corresponding DNG layer may be considered as another reactive impedance, but with an opposite sign (since the permittivity and permeability of the DNG layer have opposite sign to those of the DPS layer) [54]. Therefore, pairing these two reactive "impedances" with opposite signs may produce the conditions for a resonance. Furthermore, reducing the thicknesses of both layers can affect the values of these equivalent impedances, but their signs stay opposite. As a result, the DPS-DNG pair can still remain resonant, as we reduce their thickness while maintaining a certain ratio of thicknesses (although the bandwidth of this resonance is affected as the size is reduced). This feature is not present for a pair of conventional DPS-DPS thin layers, and it is due to juxtaposing DPS and DNG layers (and, similarly, also for ENG and MNG layers). Such pairing of complementary materials can provide us with the possibility of having sub-wavelength "compact resonant structures" in the form of sub-wavelength cavities, waveguides, and scatterers. This can be extended to the cylindrical and spherical geometries formed by pairs of complementary MTMs, as studied by Alù and Engheta [73], [74], [85], who have theoretically shown how suitable pairs of two concentric spherical shells made of DPS-DNG, ENG-MNG, or even of ENG-DPS or MNG-DPS materials may lead to significant enhancement of wave scattering when compared with scattering from structures with the same shape and dimensions, but made of standard DPS media only. In other words, these structures are indeed electrically tiny scatterers with much larger scattering cross section than ordinary scatterers of the same size. With no loss, this enhancement can be of several orders of magnitude when compared with scatterers of comparable dimensions made by standard dielectrics. This result is consistent with the phenomenon of surface plasmon resonance in nanoparticles made of noble metals [124]. These resonant structures are briefly reviewed here.

Consider a spherical structure composed of two concentric shells made of DPS and DNG materials with radii $a_{1}, a$, surrounded by free space (with permittivity $\varepsilon_{0}$ and permeability $\mu_{0}$ ) (Fig. 9). A monochromatic incident plane wave illuminates this structure. As is well known, the peaks in the scattering coefficients for such a structure are due to the excitation of the natural modes (i.e., material polaritons) supported by the structure. In other words, if at a given frequency the scatterer supports a natural mode, its scattering cross section will show a resonant peak. At a fixed frequency, this can be explored by varying the total dimension of the scatterer until we achieve the case for which a natural mode may be excited. For spherical structures made of conventional media, the presence of such modes has a cutoff dimension for the outer radius $a$ (usually comparable with the wavelength of operation) below which no natural mode is supported and, thus, the scattering coefficients are low. On the other hand, when DNG or SNG MTMs are combined with complementary materials, this "cutoff" limitation may be removed. Specifically, the dispersion relation for the material natural modes supported by the concentric-shell structure in Fig. 9 has been obtained in general [85], and in the limit of small radii, it can be simplified as

$$
\begin{aligned}
& \left\{\left(\frac{a_{1}}{a}\right)^{2 n+1}\right. \\
& \left.-\frac{\left[(n+1) \mu_{0}+n \mu_{\mathrm{DNG}}\right]\left[(n+1) \mu_{\mathrm{DNG}}+n \mu_{\mathrm{DPS}}\right]}{n(n+1)\left(\mu_{\mathrm{DNG}}-\mu_{0}\right)\left(\mu_{\mathrm{DNG}}-\mu_{\mathrm{DPS}}\right)}\right\} \\
& \times\left\{\left(\frac{a_{1}}{a}\right)^{2 n+1}\right. \\
& \left.\quad-\frac{\left[(n+1) \varepsilon_{0}+n \varepsilon_{\mathrm{DNG}}\right]\left[(n+1) \varepsilon_{\mathrm{DNG}}+n \varepsilon_{\mathrm{DPS}}\right]}{n(n+1)\left(\varepsilon_{\mathrm{DNG}}-\varepsilon_{0}\right)\left(\varepsilon_{\mathrm{DNG}}-\varepsilon_{\mathrm{DPS}}\right)}\right\}=0
\end{aligned}
$$

where $n$ is the spherical modal order of the natural mode (referring to the variation along $\theta$ ). In order to have a physical solution for $a_{1} / a$ satisfying the above equation, one or both of the expressions above containing the material permittivities or permeabilities should attain a value between zero and unity. This cannot be achieved if the concentric shells are all made of conventional DPS materials, implying that no natural mode is supported for electrically tiny spherical shells with conventional materials. However, if we use a DNG or SNG layer combined with a DPS or another properly chosen complementary MTM concentric layer, it will become possible to have a solution for $a_{1} / a$. As a result, for a specific ratio of radii $a_{1} / a$ fulfilling the above equation, the condition for the presence of a natural mode for the tiny DPS-DNG (or ENG-MNG, DPS-ENG, or DPS-MNG) concentric shells exists, which depends only on the ratio of shell radii without any direct constraint on the outer dimension of the scatterer. This behavior gives rise to the possibility of having a very high scattered field from a very tiny concentric shell particle [73], [74], [85].

Fig. 10 shows, as an example, the behavior of the scattering coefficient $c_{\mathrm{TM}}^{1}$ for the $n=1$ mode, i.e., the dipolar scattered spherical TM wave, for a case where a DPS material is covered with an ENG layer. Comparison with the DPS-DPS case is also shown in Fig. 10, which reveals a major enhancement of the scattering phenomenon. We note from Fig. 10(A) that the 


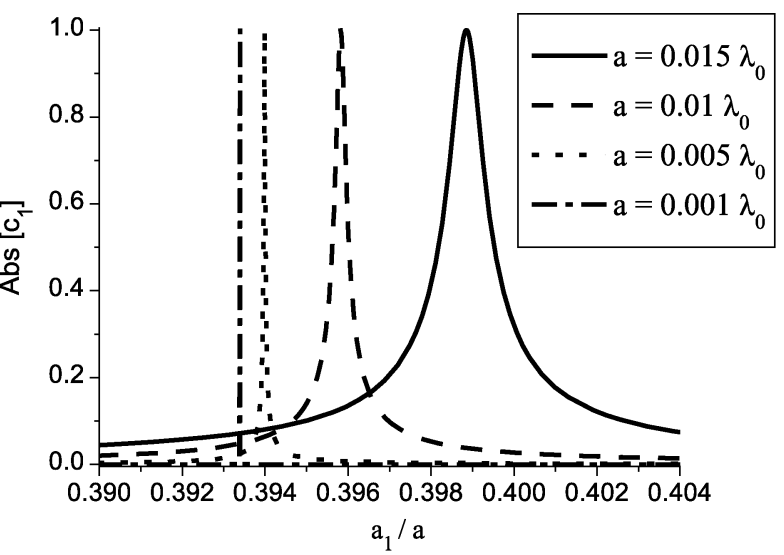

(A)

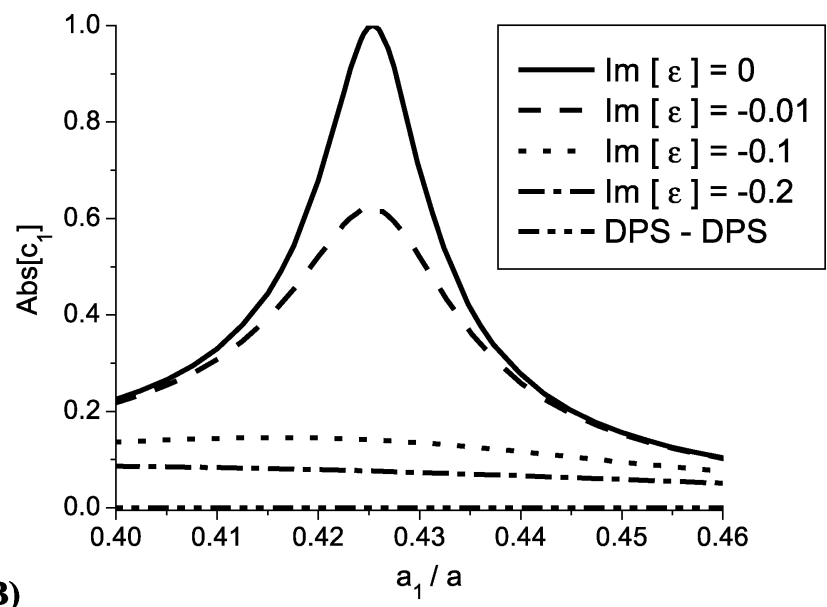

Fig. 10. Behavior of the scattering coefficient $c_{\mathrm{TM}}^{1}$ versus $a_{1} / a$ for a DPS-ENG combination with $\mu_{1}=\mu_{2}=\mu_{0}, \varepsilon_{1}=10 \varepsilon_{0}, \varepsilon_{2}=$ $\left[-1.5-j \operatorname{Im}\left(\varepsilon_{2 r}\right)\right] \varepsilon_{0} . \operatorname{In}(\mathrm{A}), \operatorname{Im}\left(\varepsilon_{2 r}\right)$ is assumed zero, and the plot shows the behavior of $\left|c_{\mathrm{TM}}^{1}\right|$ in terms of $a_{1} / a$ for several values of $a$. In (B), the effect of loss, i.e., nonzero $\operatorname{Im}\left(\varepsilon_{2 r}\right)$, is shown for $a_{1}=\lambda_{1} / 20$, where $\lambda_{1}$ is the wavelength in region 1 .

scattering coefficient can reach its maximum, even though the outer radius $a$ is much smaller than the wavelength. As the outer radius gets smaller, the maximum scattering can still occur, although it becomes much more sensitive to the ratio $a_{1} / a$. When the loss is included, the peak of the scattering coefficient is decreased as expected; however, it may still be greater than the scattering from the corresponding DPS case, as shown in Fig. 10(B).

One can speculate that by embedding these highly polarizable DPS-DNG spherical particles in a host medium, a bulk composite material with effective permittivities and/or permeabilities exhibiting resonances can be formed [85]. The resulting effective material parameters can be strongly affected by the choice of the ratio $a_{1} / a$ and can attain negative values for a certain range of that ratio [85].

There are two other interesting features about the scattering from these resonant structures worth noting. First, the large scattering amplitude from the spherical DPS-DNG shells shown above in Fig. 10 was for the dipolar term. As is well known, higher order multipoles begin to contribute more as the size of the sphere increases. However, as studied by Alù and Engheta [86], (13) can be satisfied for different ratios of the radii for cases with $n>1$, e.g., $n=2$ for quadrupolar or $n=3$ for octopolar scattering. This implies that one can obtain a strong resonant scattering amplitude for a higher order multipole (e.g., quadrupole or octopole) while keeping the electrical size of the object small and the scattering amplitudes of the lower and higher order multipoles weak. Therefore, if the ratio of the radii is chosen judiciously, a very small spherical object made from a combination of ENG, MNG, DNG, and/or DPS layers can, in principle, strongly scatter quadrupolar or octopolar fields. It is interesting to notice that ordinarily electrically small scatterers re-radiate like small dipoles because the phase retardation within them is negligible. However, these electrically and physically small DPS-DNG two-shell spheres may scatter like a quadrupole. This can offer interesting potential applications for realizing optical nanotransmission lines by arranging these nanoshells into linear arrays to transport optical energy below diffraction limits and to act as nanoantennas with quadrupole and higher order multipole radiation patterns. The second interesting feature for such DNG-DPS scatterers involves the opposite effect, the possibility of reducing the total scattering cross section of a structure. It has been suggested by Alù and Engheta [87] that, for a different ratio of radii, a "transparency" condition may be achieved, which results in the reduction of the total scattering cross section of these scatterers. In the case of thin spherical scatterers, their total cross section is generally dominated by the dipolar term in the multipole expansion. This dipolar scattering may vanish with a proper choice of the two-shell radii, and the total scattering cross section can thus be reduced. When larger scatterers are considered, this overall reduction is less effective because the multiple contributions from different multipole terms contribute more to the overall scattering cross section and cannot all be reduced simultaneously. Nonetheless, a noticeable effect is still present even in this scenario for certain proposed geometries.

Similar features have been obtained for the case of thin cylindrical structures formed by coaxial DPS and DNG (or ENG-MNG) layers [73]. For the small radii approximation, the dispersion relation for the natural modes in such cylindrical DPS-DNG structures can be expressed as

$$
\begin{aligned}
& \left\{\left(\frac{a_{1}}{a}\right)^{2 n}-\frac{\mu_{\mathrm{DNG}}+\mu_{o}}{\mu_{\mathrm{DNG}}-\mu_{o}} \frac{\mu_{\mathrm{DNG}}+\mu_{\mathrm{DPS}}}{\mu_{\mathrm{DNG}}-\mu_{\mathrm{DPS}}}\right\} \\
& \quad \times\left\{\left(\frac{a_{1}}{a}\right)^{2 n}-\frac{\varepsilon_{\mathrm{DNG}}+\varepsilon_{o}}{\varepsilon_{\mathrm{DNG}}-\varepsilon_{o}} \frac{\varepsilon_{\mathrm{DNG}}+\varepsilon_{\mathrm{DPS}}}{\varepsilon_{\mathrm{DNG}}-\varepsilon_{\mathrm{DPS}}}\right\}=0 .
\end{aligned}
$$

Again, when the ratio of radii $a_{1} / a$ satisfies this equation, the scattering amplitude reaches its maximum. Other characteristics of these cylindrical structures are discussed in [73].

\section{DNG Metamaterials AND ANTENNA APPlications}

We mentioned that pairing DNG and DPS materials (or pairing complementary SNG materials) can be regarded as joining two reactive impedances with opposite signs, resulting in a resonance phenomenon. One can then ask the following question: Can a DNG (or an SNG) layer be used to modify 
(A)

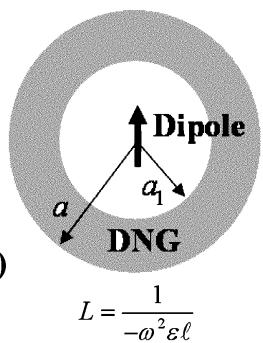

(B)

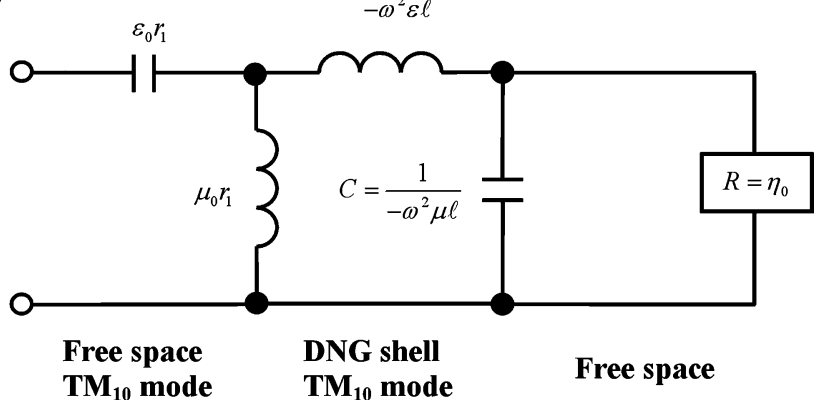

Fig. 11. (A) Electrically small electric dipole surrounded by a DNG shell. (B) Lumped-element circuit for such an antenna radiating a $\mathrm{TM}_{10}$ mode into a free-space sphere of radius $a_{1}$ that is surrounded by a lossless DNG spherical shell of thickness $\ell=a-a_{1}$, which is then terminated with a free-space region.

the input impedance of an antenna, providing the possibility to improve the antenna performance? This problem has been studied analytically and numerically by Ziolkowski and Kipple [68]. They have considered the possibility of matching an electrically small electric dipole to free space by surrounding it with a DNG shell, as shown in Fig. 11(A), and have successfully demonstrated that the dipole-DNG shell system produces large radiated power for an electrically small antenna [68].

The complex power generated by an electrically small electric dipole with current moment $I_{0} \ell$ centered in a spherical region of radius $a$ filled with a homogeneous DPS medium is well known [125]

$$
P_{\text {Dipole }}=\eta \frac{\pi}{3}\left|\frac{I_{0} l}{\lambda}\right|^{2}\left[1-\frac{j}{(k a)^{3}}\right] .
$$

When it is compared to those generated by that antenna when it is embedded in a DNG medium, it is observed that, while the radiated power in both cases was equal, the reactive power in the DNG case was equal in magnitude, but opposite in sign to the free space (DPS) case, i.e., the complex powers $P_{\text {Dipole,DPS }}=$ $P_{\mathrm{Dipole}, \mathrm{DNG}}^{*}$. This property stimulated the investigation into the effect of placing a DNG shell around an electrically small electric dipole in an attempt to "match" the dipole to free space.

An approximate lumped-element circuit model of the dipole-DNG shell system confirmed that it was possible to treat the presence of the DNG shell as a matching network. This model is shown in Fig. 11(B). The fundamental $\mathrm{TM}_{10}$ spatial mode radiated by the dipole into free space sees a high-pass filter and, thus, is basically in a cutoff situation. Normally, this mode sees a potential barrier and tunnels through it to the receiver, leaving a large amount of reactive power behind. The DNG shell produces a low-pass system that can compensate for the high-pass behavior. The DNG shell provides a way to match the corresponding $C L$ and $L C$ resonances to produce a system that is impedance matched to free space, i.e., by

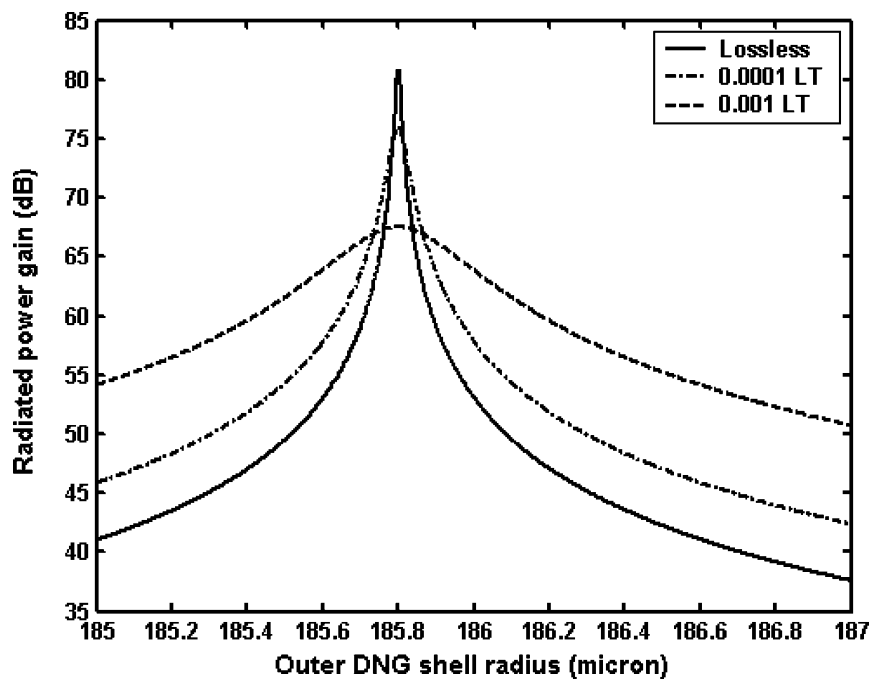

Fig. 12. Radiated power gain for the dipole-DNG shell system is considerable even in the presence of losses.

joining two regions with reactive impedances of opposite signs (capacitive for the DPS sphere and inductive for the DNG shell), the total reactance can be reduced to zero. The potential barrier and, consequently, the reactive power near the antenna are reduced to zero allowing the $\mathrm{TM}_{10}$ to propagate freely into free space. This matched-resonance source-DNG shell description is reciprocal to Alù and Engheta's resonant scattering arguments and is consistent with the 2-D planar MTM realizations considered by Eleftheriades et al. [43] and Caloz and Itoh [40]. The problem of an infinitesimal electric dipole enclosed within a DNG shell was solved analytically, and numerical evaluations of a variety of DNG cases were provided [68]. These results confirmed that the dipole-DNG shell system caused the radiated power to be increased by orders of magnitude with a simultaneous decrease in the reactance and a corresponding decrease in the radiation $Q$ to values below the Chu limit. Moreover, this behavior does not disappear in the presence of losses. Rather, since it is a resonance-based effect, losses broaden the resonance and decrease the peak of the response. The results for the "super-gain" case in [68] for which the DNG shell has $\left(\varepsilon_{\mathrm{DNG}}, \mu_{\mathrm{DNG}}\right)=\left(-3 \varepsilon_{o},-3 \mu_{o}\right)$ and an inner radius $a_{1}=100 \mu \mathrm{m}$ are shown in Fig. 12. The dipole length was assumed to be $100 \mu \mathrm{m}$. The radiated power gain, the power radiated by the dipole-DNG shell system relative to the power radiated in free space by a dipole whose half-length equals the outer radius of the DNG shell, is plotted against the outer radius of the shell. The peak of this radiated power gain occurs for an outer radius of $a=185.8 \mu \mathrm{m}$. The lossless case is compared to the lossy cases with the loss tangent $=\varepsilon_{\text {imag }} / \varepsilon_{\text {real }}=\mu_{\text {imag }} / \mu_{\text {real }}$ of the DNG MTM being 0.0001 and 0.001 . It illustrates the expected behavior. Despite the presence of losses, the radiated power gain is substantial.

The natural question arose as to the relationship between the enhanced source and scattering results. If reciprocity holds, there should be a one-to-one correspondence between a particular enhanced DPS-DNG scattering configuration and the corresponding enhanced DNG dipole-shell radiating system. To 


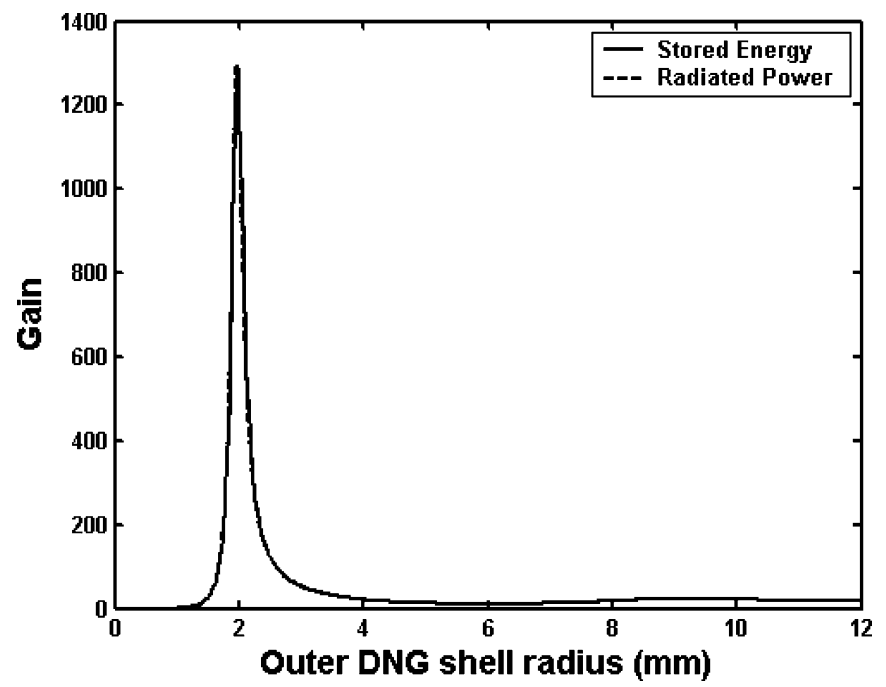

Fig. 13. For a DNG shell of inner radius $1.0 \mathrm{~mm}$ and $\left(\varepsilon_{\mathrm{DNG}}, \mu_{\mathrm{DNG}}\right)=$ $\left(-3 \varepsilon_{o},-3 \mu_{o}\right)$, the gain in the energy stored in the inner DPS sphere when a $T M_{r}(n=1, m=1)$ wave is scattered from the shell and the gain the power radiated from an electrically small dipole antenna center in the shell are given as a function of the outer radius of the shell. The correlation between these two quantities is clearly noticeable.

verify that the reciprocity holds for these DNG and ENG-MNG systems, Kipple and Ziolkowski [88] have also considered several very general sphere scattering problems both analytically and numerically. In particular, plane-wave scattering from a sphere that was coated with two concentric spherical shells, which could be DPS, DNG, ENG or MNG materials, was analyzed. The coated spheres were considered to be located in a DPS medium, i.e., free space. This allowed a direct comparison with the concentric ENG-MNG and DPS-DNG sphere results [85], a direct comparison of the free-space sphere embedded in a DNG shell with the reciprocal DNG dipole-shell system, and other interesting combinations of DPS, DNG, ENG, and MNG shells. It was verified that reciprocity holds for all of the configurations studied. To demonstrate this behavior, consider the "manufacturable" case of [68] in which an electrically small (1.2-mm length) electric dipole is embedded in a small sphere of free space with a radius of $1.0 \mathrm{~mm}$, that is, in turn, surrounded by a DNG shell having permittivity and permeability values $\left(\varepsilon_{\mathrm{DNG}}, \mu_{\mathrm{DNG}}\right)=\left(-3 \varepsilon_{o},-3 \mu_{o}\right)$. The external region is assumed to be free space, and the radiation frequency is $10 \mathrm{GHz}$. The radiated power gain, here, the ratio of the power radiated by the dipole in the presence of the DNG shell and in free space, is plotted in Fig. 13 as a function of the DNG shell's outer radius. The peak of this radiated power gain occurs for an outer radius of $a=1.966 \mathrm{~mm}$. The energy stored gain, when a $T M^{r}(n=1, m=1)$ wave is incident on these nested spheres, is also shown in Fig. 13. The gain is the ratio of the energy stored in the inner DPS (free space) sphere in the presence of the DNG shell and in the same sphere when all the regions are free space. The peak in the stored energy gain occurs at the same outer DNG shell radius, as does the peak in the radiated power gain.

A strong correlation is observed between all of the corresponding radiation and scattering results. It has also been demonstrated that the scattering resonance occurs where the stored energy in the inner sphere associated with the real part of the electric field goes to zero and with the magnetic field goes to a maximum. This behavior is correlated to an inductive reactive power; it cancels the capacitive reactive electric power associated with the dipole formed by the scattering from the electrically small shell. As a consequence of the demonstrated reciprocity between a pair of DPS-DNG and ENG-MNG shells, Kipple and Ziolkowski have also considered using only an ENG shell to produce the necessary inductive reactive power needed to cancel the capacitive reactive power of the electrically small dipole. This dipole-ENG shell system has been shown to produce a radiated power gain similar to the dipole-DNG shell system. It may be much simpler to realize physically than a dipole-DNG system.

\section{Dispersion Compensation in a Transmission Line USING DNG MTMs}

Another interesting potential application of DNG MTM is in its possible use for dispersion compensation. Cheng and $\mathrm{Zi}$ olkowski have considered the use of volumetric DNG MTMs for the modification of the propagation of signals along a microstrip transmission line [89]. If one could compensate for the dispersion along such transmission lines, signals propagating along them would not become distorted. This could lead to a simplification of the components in many systems. Microstrip dispersion can be eliminated by correcting for the frequency dependence of the effective permittivity associated with this type of transmission line. As shown in [126] and [127], for a microstrip transmission line of width $w$ and a conventional dielectric substrate height $h$, one has the approximate result for the effective relative permittivity of the air-substrate-microstrip system

$$
\varepsilon_{\mathrm{eff}}(f)=\varepsilon_{r}-\frac{\varepsilon_{r}-\varepsilon_{e s}}{1+G\left(\frac{f}{f_{d}}\right)^{2}}
$$

where the constants $f_{d}=\left(Z_{c} / 2 \mu_{0} h\right) G=$ $0.6+0.0009 Z_{c}$, the characteristic impedance $Z_{c} \cong(1 / 2 \pi) \sqrt{\left(\mu_{0} / \varepsilon_{e s} \varepsilon_{0}\right)} \log \left[F_{1}(h / w)+\sqrt{1+(2(h / w))^{2}}\right]$ with $F_{1}=6+(2 \pi-6) \exp \left[-(30.666 h / w)^{0.7528}\right]$ and the electrostatic relative permittivity $\varepsilon_{e s} \cong$ $\left(\left(\varepsilon_{r}+1\right) / 2\right)+\left(\left(\varepsilon_{r}-1\right) / 2\right)[1+10(h / w)]^{-a b}$ with $a=1+(1 / 49) \log \left[\left((w / h)^{4}+(w / 52 h)^{2} /(w / h)^{4}+\right.\right.$ $0.432)]+(1 / 18.7) \log \left[1+((1 / 18.1)(w / h))^{3}\right], \quad$ and $b=0.564\left(\left(\varepsilon_{r}-0.9 / \varepsilon_{r}+3.0\right)\right)^{0.053}$

The goal is to design an MTM that can be included with the microstrip line in some manner to make it dispersionless, i.e., we want to produce a dispersion-compensated segment of transmission line. This means we want to introduce an MTM with relative permittivity $\varepsilon_{\mathrm{MTM}}$ and permeability $\mu_{\mathrm{MTM}}$ so that the overall relative permittivity and permeability of the system is

$$
\begin{aligned}
\frac{\varepsilon(f)}{\varepsilon_{0}} & =\varepsilon_{\mathrm{eff}}(f)+\varepsilon_{\mathrm{MTM}}(f) \\
\frac{\mu(f)}{\mu_{0}} & =1+\mu_{\mathrm{MTM}}(f)
\end{aligned}
$$


in such a manner that the wave impedance in the MTM remains the same as it is in the original substrate, i.e.,

$$
Z=\sqrt{\frac{\mu(f)}{\varepsilon(f)}}=Z_{0} \sqrt{\frac{1+\mu_{\mathrm{MTM}}(f)}{\varepsilon_{\mathrm{MTM}}(f)+\varepsilon_{\mathrm{eff}}(f)}}=Z_{0} \sqrt{\frac{1}{\varepsilon_{\mathrm{eff}}(f)}}
$$

and the index of refraction in the medium compensates for the dispersion effects associated with the microstrip geometry itself, i.e., the effective index becomes that of free space

$$
\begin{aligned}
n_{\mathrm{eff}}(f)= & \sqrt{\varepsilon_{\mathrm{eff}}(f)}+\sqrt{\frac{\varepsilon(f)}{\varepsilon_{0}}} \sqrt{\frac{\mu(f)}{\mu_{0}}} \\
= & \sqrt{\varepsilon_{\mathrm{eff}}(f)}+\sqrt{\varepsilon_{\mathrm{eff}}(f)+\varepsilon_{\mathrm{MTM}}(f)} \\
& \times \sqrt{1+\mu_{\mathrm{MTM}}(f)} \\
= & 1 .
\end{aligned}
$$

These conditions are satisfied if $\varepsilon_{\text {eff }}(f)\left[1+\mu_{\mathrm{MTM}}(f)\right]=$ $\varepsilon_{\mathrm{MTM}}(f)+\varepsilon_{\mathrm{eff}}(f)$ so that

$$
\begin{aligned}
\mu_{\mathrm{MTM}}(f) & =\frac{1}{\sqrt{\varepsilon_{\text {eff }}(f)}}-1 \\
\varepsilon_{\mathrm{MTM}}(f) & =\varepsilon_{\text {eff }}(f) \mu_{\mathrm{MTM}}(f) .
\end{aligned}
$$

We note that the effective permittivity and permeability of such an MTM should be negative, implying that a DNG material must be utilized for this purpose. [The range of validity of condition (20) should be consistent with that of the effective medium approximation (16).] A plot of the index of refraction of the uncompensated line, of the MTM compensator, and of the dispersion-compensated line is shown in Fig. 14 for a microstrip transmission line at $10 \mathrm{GHz}$ using Roger's Duroid 5880 substrate. The substrate had the relative permittivity $\varepsilon_{r}=2.2$ and its height was $h=31 \mathrm{mil}=0.7874 \mathrm{~mm}$ The width of the transmission line was $w=2.428 \mathrm{~mm}=95.6 \mathrm{mil}$ to achieve a $50-\Omega$ impedance. As shown in Fig. 14, in principle, complete dispersion compensation is theoretically possible.

\section{MTMs Other THAN DNG MEDIA}

Although the focus of this paper is on the DNG MTMs, there are other classes of MTMs that can exhibit equally exciting and interesting features. Here, we briefly mention some of these media.

\section{A. MTMs With Near-Zero Refractive Index}

MTMs, in which the permittivity and/or permeability are near zero and, thus, the refractive index is much smaller than unity, can offer exciting potential applications. Planar MTMs that exhibit both positive and negative values of the index of refraction near zero have been realized experimentally by several research groups [39]-[48]. Within these studies, there have also been several demonstrations, both theoretically and experimentally, of planar MTMs that exhibit a zero index of refraction within a specified frequency band. In particular, by matching the resonances in a series-parallel lumped-element circuit realization of a DNG MTM at a specified frequency, the propa-

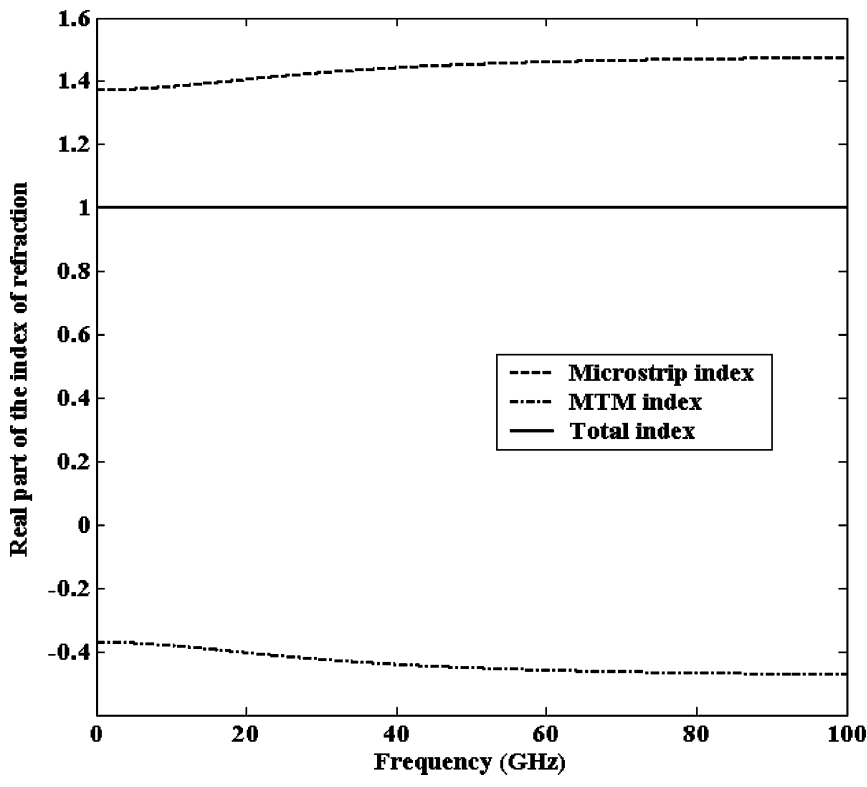

Fig. 14. Real part of the index of refraction of the microstrip-only, of the MTM-only, and the total MTM-dispersion-compensated transmission line.

gation constant as a function of frequency continuously passes through zero (giving a zero index) with a nonzero slope (giving a nonzero group speed) in its transition from a DNG region of its operational behavior to a DPS region [40], [43], [115]. Several applications of these series-parallel MTMs have been proposed and realized, e.g., phase shifters, couplers, and compact resonators.

Several investigations have also presented volumetric MTMs that exhibit near-zero-index medium properties, e.g., [49]-[53]. These zero-index EBG structure studies include working in a passband. By introducing a source into a zero-index EBG with an excitation frequency that lies within the EBG's passband, Enoch et al. [51], [52] and Tayeb et al. [53] produced an extremely narrow antenna pattern.

Alù $e t$ al. have also shown theoretically that by covering a sub-wavelength tiny aperture in a flat perfectly conducting screen with a slab of materials with $\mu \ll \mu_{o}$, one can significantly increase the power transmitted through such a hole due to the coupling of the incident wave into the "leaky" wave supported by such a layer [90]. By covering both sides of the hole, not only can one increase the transmitted power through the hole, but this power can be directed as a sharp beam in a given direction [90], [91].

These results stimulated a study by Ziolkowski [92] that details the propagation and scattering properties of a passive dispersive MTM that is matched to free space and has an index of refraction equal to zero. 1-D, 2-D, and 3-D problems corresponding to source and scattering configurations have been treated analytically. The 1-D and 2-D results have been confirmed numerically with FDTD simulations. It has been shown that the electromagnetic fields in a matched zero-index medium (i.e., $\varepsilon_{\text {real }}\left(\omega_{0}\right) \cong 0, \mu_{\text {real }}\left(\omega_{0}\right) \cong 0$ so that $Z\left(\omega_{0}\right)=Z_{0}$ and $\left.n_{\text {real }}\left(\omega_{0}\right) \cong 0\right)$ take on a static character in space, yet remain dynamic in time in such a manner that the underlying physics remains associated with propagating fields. Zero phase variation at various points in the zero-index medium has been demon- 
(A)

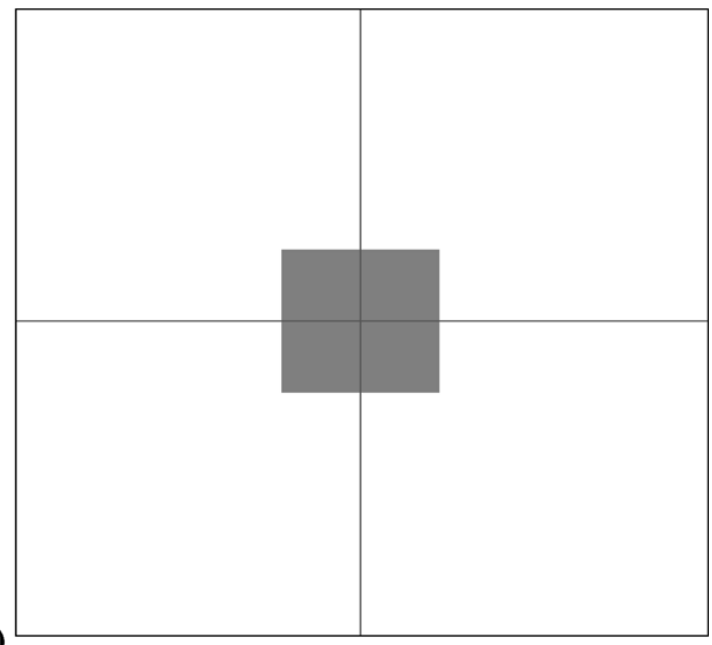

(B)

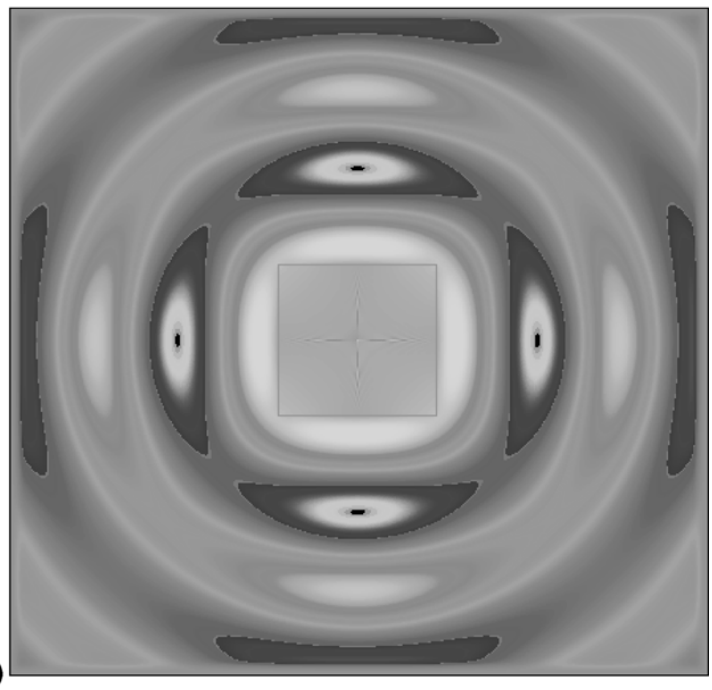

Fig. 15. (A) Line source is located at the center of a matched zero-index square cylinder of Drude medium with side length $1.2 \lambda_{o}$. (B) Electric-field intensity produced by such a line source driven at $30 \mathrm{GHz}$.

strated once steady-state conditions are obtained. These behaviors have been used to illustrate why a zero-index MTM, such as a zero-index electromagnetic bandgap structured medium, significantly narrows the far-field pattern associated with an antenna located within it. The geometry and FDTD results for a line source centered in a square matched zero-index cylinder of side length $1.2 \lambda_{0}$ and driven at $30 \mathrm{GHz}$ are shown in Fig. 15. The uniformity of the constant electric field over the entire interior of the square cylinder is clearly seen. Moreover, one can see that the fields radiated into free space arise locally as though they are driven by uniform fields across apertures corresponding to the sides of the square.

There may be a variety of potential applications for matched zero-index media beyond their already demonstrated use for compact resonators and highly directive sources and apertures. These include delay lines with no phase differences between their inputs and outputs and wavefront transformers, i.e., a transformer that converts wavefronts with small curvature into output beams with large curvature (planar) wavefronts. Other MTMs could be designed by engineering the permittivity and permeability models to yield a matched zero-index medium with a tailored wave speed. Complete spatio-temporal wavefront engineering could then be realized. Many of these issues are currently being investigated.

\section{B. MTMs as Artificial Magentic Conductors}

If an MTM can be engineered to possess a large permeability, it will behave as a "magnetic conductor." Several planar and volumetric MTM structures have been investigated that act as AMCs, i.e., slabs that produce reflection coefficient with zero phase, i.e., an in-phase reflection [93]-[99]. It has been shown by Erentok et al. [100] that a volumetric MTM constructed from a periodic arrangement of capacitively loaded loops (CLLs) acts as an AMC when the incident wave first interacts with the capacitor side of the CLLs and as a perfect electric conductor (PEC) from the opposite direction. The CLL MTM has effective material properties that exhibit a two-time-derivative Lorentz material (2TDLM) behavior for the permeability and a Drude behavior for the permittivity. The resonance of the real part of the 2TDLM model and the zero crossing of the real part of the Drude model occur at the same frequency at which the in-phase reflection occurs. This concurrence of the critical frequencies of both models produces an MTM slab with a high-impedance state at that frequency, i.e., $\lim _{\omega \rightarrow \omega_{0}} \sqrt{\mu(\omega) / \varepsilon(\omega)} \rightarrow \infty$. Numerical simulation and experimental results for the CLL-based MTM slab have shown good agreement.

The use of the two-CLL-deep MTM AMC block for antennas was also considered [100]. Numerical simulations of the interaction of a dipole antenna with such an MTM block have shown the expected AMC enhancements of the radiated fields. The dipole-AMC block configuration is shown in Fig. 16(A). The behavior of this system as a function of the antenna length $\ell$ and the distance of the antenna from the block $h$ have been studied. As shown in Fig. 16(B), it was found that resonant responses are obtained when the distance between the dipole and MTM block was optimized. Significantly enhanced electric-field values in the reflected field region and front-to-back ratios have been demonstrated. The $E$ - and $H$-plane patterns of the dipole-AMC block system and of the free-space dipole are compared in Fig. 17 for the optimized case of a $\ell=0.325 \lambda_{0}$ antenna driven at $10 \mathrm{GHz}\left(\lambda_{0}=30 \mathrm{~mm}\right)$ near a CLL-based AMC block with dimensions $7.1 \mathrm{~mm} \times 6.6 \mathrm{~mm} \times 25.4 \mathrm{~mm}$. The broadside power is more than doubled in the presence of the AMC block. The realized front-to-back ratio, as shown in Fig. 16(B), for this case, is 164.25 .

\section{Single-Negative MTMs and Plasmonic Media}

We mentioned earlier that some of the exciting features and interesting potential applications of DNG MTMs may also be developed using SNG materials such as plasmonic media. This is particularly the case where the electrical and physical dimensions of devices and components involving these materials are small. Furthermore, as shown above, when complementary SNG materials are paired, e.g., when an ENG layer (e.g., a plasmonic layer such as silver or gold in the visible or IR regimes) is juxtaposed with an MNG one, some interesting features, which are specific to pairing of these layers and which are not present for each single layer alone, may appear [25]-[27]. One of these features, a counterpart to the lensing effect of 


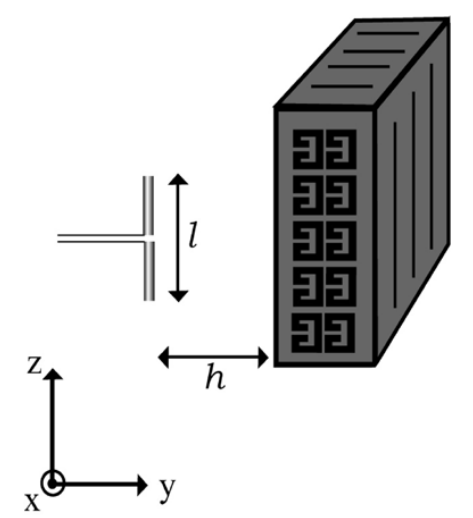

(A)

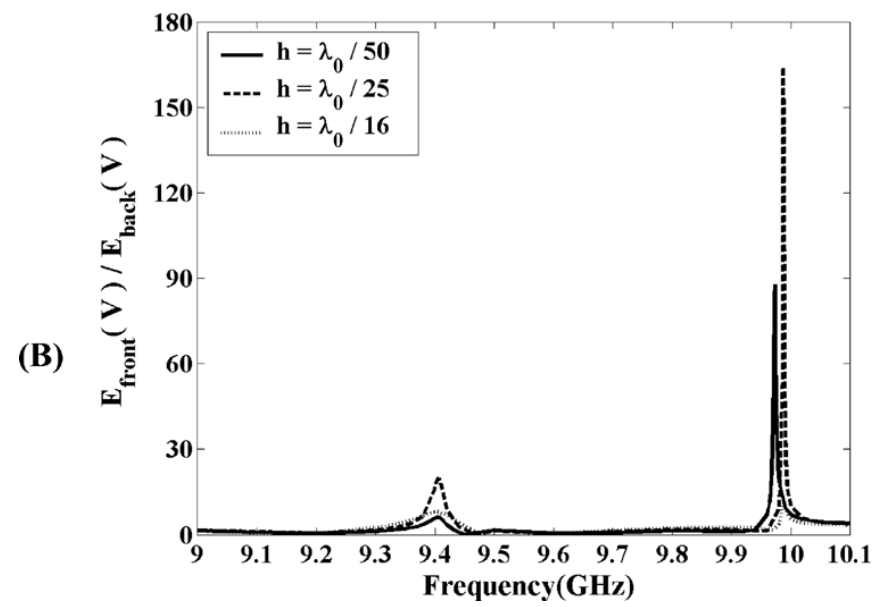

Fig. 16. (A). Dipole antenna and CLL-MTM block configuration. (B) Resonant interaction between the dipole antenna and CLL-based MTM block produces very large front-to-back ratios.

DNG slabs, is the virtual image formation, wave tunneling, growing evanescent fields, and evanescent wave displacement in a pair of ENG-MNG slabs [25]. Consider a pair of lossless ENG-MNG slabs, in front of which a point (or a line) source of monochromatic wave is placed (Fig. 18). As is usually done, the field distribution at the object plane can, in general, be expanded in terms of all spatial Fourier components (propagating and evanescent parts). Alù and Engheta have theoretically found that by judiciously selecting the ENG and MNG material parameters $\varepsilon_{\mathrm{ENG}}, \mu_{\mathrm{ENG}}, \varepsilon_{\mathrm{MNG}}$, and $\mu_{\mathrm{MNG}}$ and the thicknesses $d_{\mathrm{ENG}}$ and $d_{\mathrm{MNG}}$, one can achieve a situation in which all spatial Fourier components can "tunnel" through this paired ENG-MNG structure and, thus, the pair effectively becomes "transparent" [25]. In other words, the propagating, as well as evanescent waves at the "entrance" face of this pair of slabs, can, under certain conditions, tunnel through the pair, and show up at the exit face with the same corresponding values (in both magnitude and phase) as their values at the entrance face. As a result, an observer on the other side of this "conjugate" matched pair of ENG-MNG slabs will see a "virtual" image of the point (or line) source as though it were seated closer to the observer by the amount $d_{\mathrm{ENG}}+d_{\mathrm{MNG}}$ providing near-field observation of the objects with ideally all spatial Fourier components present [25]. This effect may conceptually provide an interesting future application in image reconstruction, resolution

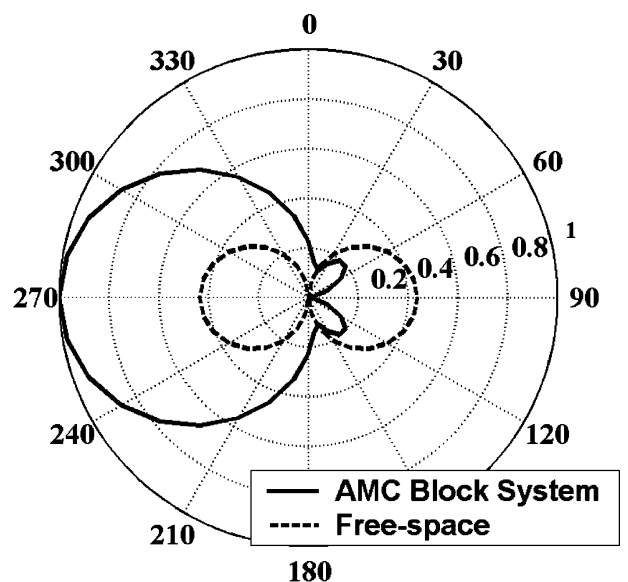

A. E-plane pattern

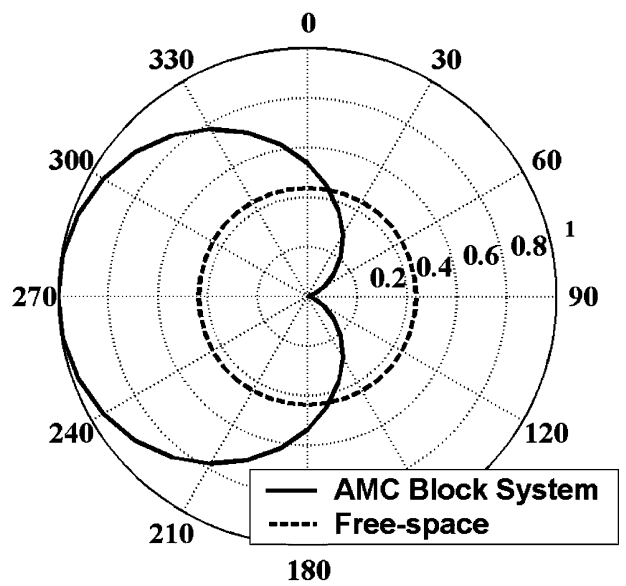

B. H-plane pattern

Fig. 17. Far-field patterns of the dipole antenna and CLL-based MTM block system (solid line), shown in Fig. 16, are compared to those produced by a free-space dipole antenna (dotted line). (A) $E$-plane pattern. (B) $H$-plane pattern.

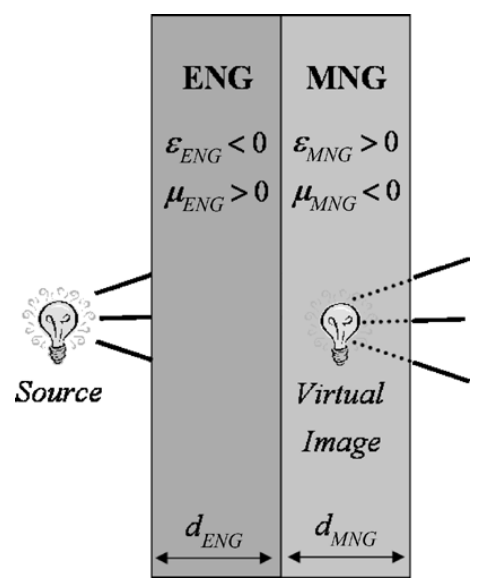

Fig. 18. Pair of ENG-MNG layers under certain conditions may provide image displacement and virtual image reconstruction. From [25].

enhancement, near-field subwavelength imaging, and NSOM. It is important to point out that an analogous matched pairing of DNG and DPS slabs would also "preserve" and allow "tunneling" of the evanescent waves, analogous to what Pendry has found for his DNG lens surrounded by a conventional medium. However, Pendry's lens ideally forms a "real" image of the 
object, whereas this ENG-MNG bilayer may conceptually displace a "virtual" image [25]. The field distributions inside such an ENG-MNG bilayer have been thoroughly analyzed, and using equivalent transmission-line models with appropriate distributed series and shunt reactive elements, various effects such as growing evanescent fields at the ENG-MNG interface, tunneling and transparency have been explained and physically justified [25]. Numerical simulations for some of these features are currently being conducted [101].

\section{EPILOGUE}

We have tried to share with you a wide variety of physical effects associated with DNG and SNG MTMs and their potential applications. While the physics of MTMs appears to be much better understood now through analysis and numerical simulations, there are significant challenges ahead in the areas of fabrication and measurements. There have been several successful microwave realizations of the volumetric MTMs that have demonstrated the unusual properties discussed here. However, since the required inclusion size is much smaller than a wavelength for these MTMs, the move to millimeter, terahertz, IR, and visible frequencies will require the development of a host of innovative structures and fabrication processes. Nonetheless, the ability to tailor material properties to achieve physical effects not thought to be possible only a few years ago is motivating a large number of activities in these directions. The future is indeed very positive for DNG MTMs.

\section{ACKNOWLEDGMENT}

The authors would like to thank their graduate students at the University of Pennsylvania, Philadelphia, and the University of Arizona, Tucson, respectively, for all their contributions to the MTM research activities in their groups. For the materials presented here, the authors particularly thank A. Alù, University of Pennsylvania and Universita di Roma Tre, Rome, Italy, Allison Kipple, University of Arizona and U. S. Army Electronic Proving Ground, Ft. Huachuca, AZ, and Aycan Erentok, University of Arizona.

\section{REFERENCES}

[1] V. G. Veselago, "The electrodynamics of substances with simultaneously negative values of $\varepsilon$ and $\mu$," Sov. Phys._Usp., vol. 47, pp. 509-514, Jan.-Feb. 1968

[2] — , "The electrodynamics of substances with simultaneously negative values of $\varepsilon$ and $\mu$ " (in Russian), Usp. Fiz. Nauk, vol. 92, pp. 517-526, 1967.

[3] J. B. Pendry, A. J. Holden, D. J. Robbins, and W. J. Stewart, "Magnetism from conductors and enhanced nonlinear phenomena," IEEE Trans. Microw. Theory Tech., vol. 47, no. 11, pp. 2075-2081, Nov. 1999.

[4] — , "Low-frequency plasmons in thin wire structures," J. Phys., Condens. Matter, vol. 10, pp. 4785-4809, 1998.

[5] D. R. Smith, W. J. Padilla, D. C. Vier, S. C. Nemat-Nasser, and S. Schultz, "Composite medium with simultaneously negative permeability and permittivity," Phys. Rev. Lett., vol. 84, pp. 4184-4187, May 2000.

[6] D. R. Smith and N. Kroll, "Negative refractive index in left-handed materials," Phys. Rev. Lett., vol. 85, pp. 2933-2936, Oct. 2000.

[7] R. A. Shelby, D. R. Smith, S. C. Nemat-Nasser, and S. Schultz, "Microwave transmission through a two-dimensional, isotropic, left-handed metamaterial," Appl. Phys. Lett., vol. 78, pp. 489-491, Jan. 2001.

[8] A. Shelby, D. R. Smith, and S. Schultz, "Experimental verification of a negative index of refraction," Science, vol. 292, pp. 77-79, Apr. 2001.

[9] IEEE Trans. Antennas Propag. (Special Issue), vol. 51, no. 10, Oct. 2003.
[10] J. B. Pendry and D. Smith, "Reversing light with negative refraction," Phys. Today, vol. 57, pp. 37-43, Jun. 2004.

[11] Opt. Express (Focus Issue), vol. 11, no. 7, pp. 639-760, Apr. 2003.

[12] R. W. Ziolkowski and E. Heyman, "Wave propagation in media having negative permittivity and permeability," Phys. Rev. E, Stat. Phys. Plasmas Fluids Relat. Interdiscip. Top., vol. 64, Oct. 2001. Paper 056625.

[13] R. W. Ziolkowski, "Design, fabrication, and testing of double negative metamaterials," IEEE Trans. Antennas Propag., vol. 51, no. 7, pp. 1516-1529, Jul. 2003.

[14] P. M. Valanju, R. M. Walter, and A. P. Valanju, "Wave refraction in negative-index media: Always positive and very inhomogeneous," Phys. Rev. Lett., vol. 88, 2002. Paper 187401.

[15] D. R. Smith, D. Schurig, and J. B. Pendry, "Negative refraction of modulated electromagnetic waves," Appl. Phys. Lett, vol. 81, no. 15, pp. 2713-2715, Oct., 72002.

[16] I. V. Lindell, S. A. Tretyakov, K. I. Nikoskinen, and S. Ilvonen, "BW media-Media with negative parameters, capable of supporting backward waves," Microwave Opt. Technol. Lett., vol. 31, no. 2, pp. 129-133, 2001.

[17] M. W. McCall, A. Lakhtakia, and W. S. Weiglhofer, "The negative index of refraction demystified," Eur. J. Phys., vol. 23, pp. 353-359, 2002.

[18] A. Lakhtakia, "Reversed circular dichroism of isotropic chiral mediums with negative permeability and permittivity," Microwave Opt. Technol. Lett., vol. 33, no. 2, pp. 96-97, Apr. 20, 2002.

[19] J. C. Bose, "On the rotation of plane of polarization of electric waves by a twisted structure," Proc. R. Soc., vol. 63, pp. 146-152, 1988.

[20] I. V. Lindell, A. H. Sihvola, and J. Kurkijarvi, "Karl F. Lindman: The last Hertzian, and a harbinger of electromagnetic chirality," IEEE Antennas Propag. Mag., vol. 34, no. 3, pp. 24-30, 1992.

[21] W. E. Kock, "Metallic delay lenses," Bell Syst. Tech. J., vol. 27, pp. 58-82, 1948.

[22] W. Rotman, "Plasma simulation by artificial dielectrics and parallelplate media," IRE Trans. Antennas Propag., vol. 10, no. 1, pp. 82-95, Jan. 1962.

[23] J. Electromagn. Waves Applicat. (Special Issue), vol. 6, no. 5/6, 1992.

[24] D. R. Fredkin and A. Ron, "Effective left-handed (negative index) composite material," Appl. Phys. Lett., vol. 81, no. 10, pp. 1753-1755, Sep., 22002.

[25] A. Alù and N. Engheta, "Pairing an epsilon-negative slab with a mu-negative slab: Resonance, tunneling and transparency," IEEE Trans. Antennas Propag., vol. 51, no. 10, pp. 2558-2571, Oct. 2003.

[26] - "Guided modes in a waveguide filled with a pair of single-negative (SNG), double-negative (DNG), and/or double-positive (DPS) layers," IEEE Trans. Microw. Theory Tech., vol. 52, no. 1, pp. 199-210, Jan. 2004.

[27] _ "An overview of salient properties of guided-wave structures with double-negative and single-negative metamaterials," in Negative Refraction Metamaterials: Fundamental Properties and Applications, G. V. Eleftheriades and K. G. Balmain, Eds., to be published.

[28] R. B. Greegor, C. G. Parazzoli, K. Li, B. E. C. Koltenbah, and M. Tanielian, "Experimental determination and numerical simulation of the properties of negative index of refraction materials," Opt. Express, vol. 11, pp. 688-695, 2003 [Online]. Available: http://www.opticsexpress.org/abstract.cfm?URI=OPEX-11-7-688.

[29] R. B. Greegor, C. G. Parazzoli, K. Li, and M. H. Tanielian, "Origin of dissipative losses in negative index of refraction materials," Appl. Phys. Lett., vol. 82, pp. 2356-2358, 2003.

[30] C. G. Parazzoli, R. B. Greegor, K. Li, B. E. C. Koltenbah, and M. Tanielian, "Experimental verification and simulation of negative index of refraction using Snell's law," Phys. Rev. Lett., vol. 90, 2003. Paper 107401.

[31] S. Hrabar, Z. Eres, and J. Bartolic, "Capacitively loaded loop as basic element of negative permeability meta-material," in Proc. 32nd Eur. Microwave Conf., Milan, Italy, Sep. 24-26, 2002.

[32] P. Gay-Balmaz and O. J. F. Martin, "Efficient isotropic magnetic resonators," Appl. Phys. Lett., vol. 81, no. 5, pp. 939-941, Jul., 292002.

[33] R. Marques, F. Medina, and R. Rafii-El-Idrissi, "Role of bianisotropy in negative permeability and left-handed metamaterials," Phys. Rev. B, Condens. Matter, vol. 65, no. 14, 2002. Paper 144440.

[34] R. Marques, J. Martel, F. Mesa, and F. Medina, "A new 2-D isotropic left-handed metamaterial design: Theory and experiment," Microwave Opt. Technol. Lett., vol. 36, pp. 405-408, Dec. 2002.

[35] R. Marques, F. Mesa, J. Martel, and F. Medina, "Comparative analysis of edge- and broadside- coupled split ring resonators for metamaterial design-Theory and experiments," IEEE Trans. Antennas Propag., vol. 51, no. 10, pp. 2572-2581, Oct. 2003. 
[36] M. M. I. Saadoun and N. Engheta, Theoretical Study of Electromagnetic Properties of Nonlocal Omega Media, ser. PIER Monograph, A. Priou, Ed., 1994, vol. 9, ch. 15, pp. 351-397.

[37] N. Engheta, S. Nelatury, and A. Hoorfar, "The role of geometry of inclusions in forming metamaterials with negative permittivity and permeability," in Proc. XXVII URSI Gen. Assembly, Maastricht, The Netherlands, Aug. 17-24, 2002 [CD ROM], Paper 1935.

[38] J. McVay, N. Engheta, and A. Hoorfar, "Space-filling-curve elements as possible inclusions for double-negative metamaterials," in USNC-URSI Nat. Radio Science Meeting Dig., Monterey, CA, Jun. 20-26, 2004, p. 136.

[39] C. Caloz, C.-C. Chang, and T. Itoh, "Full-wave verification of the fundamental properties of left-handed materials in waveguide configurations," J. Appl. Phys., vol. 90, pp. 5483-5486, Dec. 2001.

[40] C. Caloz and T. Itoh, "Microwave applications of novel metamaterials," in Proc. Int. Electromagnetics in Advanced Applications Conf., Turin, Italy, Sep. 2003, pp. 427-430.

[41] C. Caloz, A. Sanada, and T. Itoh, "A novel composite right/left-handed coupled-line directional coupler with arbitrary coupling level and broad bandwidth," IEEE Trans. Microw. Theory Tech., vol. 52, no. 3, pp. 980-992, Mar. 2004.

[42] C. Caloz and T. Itoh, "A novel mixed conventional microstrip and composite right/left-handed backward wave directional coupler with broadband and tight coupling characteristics," IEEE Microw. Wireless Compon. Lett., vol. 14, no. 1, pp. 31-33, Jan. 2004.

[43] G. G. V. Eleftheriades, A. K. Iyer, and P. C. Kremer, "Planar negative refractive index media using periodically $L-C$ loaded transmission lines," IEEE Trans. Microw. Theory Tech., vol. 50, no. 12, pp. 2702-2712, Dec. 2002.

[44] A. A. Grbic and G. V. Eleftheriades, "Experimental verification of backward-wave radiation from a negative refractive index metamaterial," $J$. Appl. Phys., vol. 92, pp. 5930-5935, Nov. 2002.

[45] R. Islam, F. Eleck, and G. V. Eleftheriades, "Coupled-line metamaterial coupler having co-directional phase but contra-directional power flow," Electron. Lett., vol. 40, no. 5, pp. 315-317, Mar. 2004.

[46] A. Grbic and G. V. Eleftheriades, "Overcoming the diffraction limit with a planar left-handed transmission lines," Phys. Rev. Lett., vol. 92, no. 11, p. 117403 , Mar. 2004

[47] A. A. Oliner, "A periodic-structure negative-refractive-index medium without resonant elements," in IEEE AP-S Int. Symp./USNC/URSI Nat. Radio Science Meeting Dig., San Antonio, TX, Jun. 16-21, 2002, p. 41.

[48] A. A. Oliner, "A planar negative-refractive-index medium without resonant elements," in IEEE MTT-S Int. Microwave Symp. Dig., Philadelphia, PA, Jun. 8-13, 2003, pp. 191-194.

[49] B. Gralak, S. Enoch, and G. Tayeb, "Anomalous refractive properties of photonic crystals," J. Opt. Soc. Amer. A, Opt. Image Sci., vol. 17, no. 6, pp. 1012-1020, 2000.

[50] M. Notomi, "Theory of light propagation in strongly modulated photonic crystals: Refractionlike behavior in the vicinity of the photonic bandgap," Phys. Rev. B, Condens. Matter, vol. 62, no. 16, 2000. Paper 10696.

[51] S. Enoch, G. Tayeb, P. Sabouroux, N. Guerin, and P. Vincent, "A metamaterial for directive emission," Phys. Rev. Lett., vol. 89, Nov. 2002, Paper 213902.

[52] S. Enoch, G. Tayeb, and B. Gralak, "The richness of dispersion relation of electromagnetic bandgap materials," IEEE Trans. Antennas Propag., vol. 51, no. 10, pp. 2659-2666, Oct. 2003.

[53] G. Tayeb, S. Enoch, P. Vincent, and P. Sabouroux, "A compact directive antenna using ultrarefractive properties of metamaterials," in Proc. Int. Electromagnetics Advanced Applications Conf., Turin, Italy, Sep. 2003, pp. 423-426.

[54] N. Engheta, "An idea for thin subwavelength cavity resonators using metamaterials with negative permittivity and permeability," IEEE Antennas Wireless Propag. Lett., vol. 1, no. 1, pp. 10-13, 2002.

[55] J. A. Kong, B.-I. Wu, and Y. Zhang, "A unique lateral displacement of a Gaussian beam transmitted through a slab with negative permittivity and permeability," Microwave Opt. Technol. Lett., vol. 33, pp. 136-139, Mar. 2002.

[56] P. Kolinko and D. R. Smith, "Numerical study of electromagnetic waves interacting with negative index materials," Opt. Express, vol. 11, pp. 640-648, Apr. 2003.

[57] R. W. Ziolkowski, "Pulsed and CW Gaussian beam interactions with double negative metamaterial slabs," Opt. Express, vol. 11, pp. 662-681, Apr. 2003.

[58] _ - "Pulsed and CW Gaussian beam interactions with double negative metamaterial slabs: Errata," Opt. Express, vol. 11, no. 13, pp. 1596-1597, Jun. 30, 2003.
[59] S. Foteinopoulou, E. N. Economou, and C. M. Soukoulis, "Refraction in media with a negative refractive index," Phys. Rev. Lett., vol. 90, Mar. 2003. Paper 107402.

[60] N. Engheta, "Ideas for potential applications of metamaterials with negative permittivity and permeability," in Advances in Electromagnetics of Complex Media and Metamaterials. ser. NATO Sci., S. Zouhdi, A. H. Sihvola, and M. Arsalane, Eds: Kluwer, 2002, pp. 19-37.

[61] I. S. Nefedov and S. A. Tretyakov, "Waveguide containing a backward-wave slab," ArXiv.org, Nov. 2002 [Online]. Available: http://arxiv.org/pdf/cond-mat/0211185.

[62] B.-I. Wu, T. M. Grzegorczyk, Y. Zhang, and J. A. Kong, "Guided modes with imaginary transverse wave number in a slab waveguide with negative permittivity and permeability," J. Appl. Phys., vol. 93, no. 11, pp. 9386-9388, Jun. 2003.

[63] A. Topa, "Contradirectional interaction in a NRD waveguide coupler with a metamaterial slab," in XXVII Int. Union of Radio Science General Assembly, Maastricht, The Netherlands, Aug. 17-24, 2002 [CD ROM], Paper 1878.

[64] J. B. Pendry, "Negative refraction makes a perfect lens," Phys. Rev. Lett., vol. 85, pp. 3966-3969, Oct. 2000.

[65] J. Lu, T. M. Grzegorczyk, Y. Zhang, J. Pacheco, Jr., B.-I. Wu, J. A. Kong, and M. Chen, "Čerenkov radiation in materials with negative permittivity and permeability," Opt. Express, vol. 11, pp. 723-734, Apr. 2003.

[66] Z. M. Zhang and C. J. Fu, "Unusual photon tunneling in the presence of a layer with negative refractive index," Appl. Phys. Lett., vol. 80, pp. 1097-1099, Feb. 2002.

[67] L. Wu, S. He, and L. Chen, "On unusual narrow transmission bands for a multilayered periodic structure containing left-handed materials," Opt. Express, vol. 11, pp. 1283-1290, Jun. 2003.

[68] R. W. Ziolkowski and A. Kipple, "Application of double negative metamaterials to increase the power radiated by electrically small antennas," IEEE Trans. Antennas Propag., vol. 51, no. 10, pp. 2626-2640, Oct. 2003.

[69] —-, "Causality and double-negative metamaterials," Phys. Rev. E, Stat. Phys. Plasmas Fluids Relat. Interdiscip. Top., vol. 68, Aug. 2003. Paper 026615.

[70] K. G. Balmain, A. A. E. Lüttgen, and P. C. Kremer, "Resonance cone formation, reflection, and focusing in a planar anisotropic metamaterial," IEEE Antennas Wireless Propag. Lett., vol. 1, no. 7, pp. 146-149, 2002.

[71] K. G. Balmain, A. A. E. Luttgen, and P. C. Kremer, "Power flow for resonance cone phenomena in planar anisotropic metamaterials," IEEE Trans. Antennas Propag., vol. 51, no. 10, pp. 2612-2618, Oct. 2003.

[72] N. Engheta, "Is Foster's reactance theorem satisfied in double-negative and single-negative media?," Microwave Opt. Technol. Lett., vol. 39, no. 1, pp. 11-14, Oct. 2003.

[73] A. Alù and N. Engheta, "Resonances in sub-wavelength cylindrical structures made of pairs of double-negative and double-negative or epsilon-negative and mu-negative coaxial shells," in Proc. Int. Electromagnetics and Advance Applications Conf., Turin, Italy, Sep. 8-12, 2003, pp. 435-438.

[74] $\longrightarrow$, "Sub-wavelength resonant structures containing double-negative (DNG) or single-negative (SNG) media: Planar, cylindrical and spherical cavities, waveguides, and open scatterers," in Progress in Electromagnetic Research Symp., Waikiki, HI, Oct. 13-16, 2003, p. 12.

[75] S. Hrabar, J. Bartolic, and Z. Sipus, "Experimental verification of subwavelength resonator based on backward-wave metamaterials," in IEEE AP-S Int. Symp. Dig., vol. 3, Monterey, CA, Jun. 20-25, 2004, pp. 2568-2571.

[76] A. Alù and N. Engheta, "Mode excitation by a line source in a parallelplate waveguide filled with a pair of parallel double-negative and doublepositive slabs," in IEEE AP-S Int. Symp. Dig., vol. 3, Columbus, OH, Jun. 22-27, 2003, pp. 359-362.

[77] A. Alù and N. Engheta, "Distributed-circuit-element description of guided-wave structures and cavities involving double-negative or single-negative media," in Proc. SPIE, vol. 5218, San Diego, CA, Aug. 3-8, 2003, pp. 145-155.

[78] A. Alù and N. Engheta, "Anomalous mode coupling in guided-wave structures containing metamaterials with negative permittivity and permeability," in Proc. IEEE Nanotechnology, Washington, DC, Aug. 26-28, 2002, pp. 233-234.

[79] P. Baccarelli, P. Burghignoli, G. Lovat, and S. Paulotto, "Surface-wave suppression in metamaterial grounded slabs," IEEE Antennas Wireless Propag. Lett., vol. 2, no. 19, pp. 269-272, 2003.

[80] A. Alù and N. Engheta, "Anomalies in surface wave propagation along double-negative and single-negative cylindrical shells," presented at the Progress in Electromagnetic Research Symp., Pisa, Italy, Mar. 28-31, 2004. 
[81] D. R. Smith, D. Schurig, M. Rosenbluth, S. Schultz, S. Anantha Ramakrishna, and J. B. Pendry, "Limitation on subdiffraction imaging with a negative refractive index slab," Appl. Phys. Lett., vol. 82, no. 10, pp. 1506-1508, Mar. 2003.

[82] C. Luo, S. G. Johnson, and J. D. Joannopoulos, "Subwavelength imaging in photonic crystals," Phys. Rev. B, Condens. Matter, vol. 68, 2003. Paper 045115.

[83] A. Alù and N. Engheta, "A physical insight into the 'growing' evanescent fields of double-negative metamaterial lens using its circuit equivalence," IEEE Trans. Antennas Propag., submitted for publication.

[84] _ - "Tunneling and 'growing evanescent envelopes' in a pair of cascaded sets of frequency selective surfaces in their band gaps," in Proc. URSI Int. Electromagnetic Theory Symp., vol. 1, Pisa, Italy, May 24-27, 2004, pp. 90-92.

[85] A. Alù and N. Engheta, "Polarizabilities and effective material parameters for collection of spherical nano-particles containing concentric double-negative (DNG) and single-negative (SNG) shells," in Proc. URSI Int. Electromagnetic Theory Symp., vol. 1, Pisa, Italy, May 24-27, 2004, pp. 24-26.

[86] - "Strong quadrupole scattering from ultra small metamaterial nanoshells," in IEEE AP-S Int. Symp./USNC-URSI Nat. Radio Science Meeting, Monterey, CA, Jun. 20-26, 2004, p. 210.

[87] — "Reducing scattering from cylinders and spheres using metamaterials," in IEEE AP-S Int. Symp./USNC-URSI Nat. Radio Science Meeting, Monterey, CA, Jun. 20-26, 2004, p. 231.

[88] A. D. Kipple and R. W. Ziolkowski, "Resonant scattering and enhanced radiated power by an electrically small antenna in the presence of nested metamaterial shells," Phys. Rev. Lett., Jul. 2004, submitted for publication.

[89] C.-Y. Cheng and R. W. Ziolkowski, "Tailoring double negative metamaterial responses to achieve anomalous propagation effects along microstrip transmission line," IEEE Trans. Microw. Theory Tech., vol. 51, no. 12 , pp. 2306-2314, Dec. 2003.

[90] A. Alù, F. Bilotti, N. Engheta, and L. Vegni, "How metamaterials may significantly affect the wave transmission through a sub-wavelength hole in a flat perfectly conducting screen," presented at the Metamaterials for Microwave and (Sub) Millimeter Wave Applications: Photonic Bandgap and Double Negative Designs, Components and Experiments Workshop, London, U.K., Nov. 24, 2003.

[91] — "Metamaterial bilayers for enhancement of wave transmission through a small hole in a flat perfectly conducting screen," in IEEE AP-S Int. Symp. Dig., vol. 3, Monterey, CA, Jun. 20-26, 2004, pp. 3163-3166.

[92] R. W. Ziolkowski, "Propagation in and scattering from a matched metamaterial having a zero index of refraction," Phys. Rev. E, Stat. Phys. Plasmas Fluids Relat. Interdiscip. Top., vol. 70, 2004. Paper 046608.

[93] D. Sievenpiper, L. Zhang, R. F. Jimenez Broas, N. G. Alexopolous, and E. Yablonovitch, "High-impedance electromagnetic surfaces with a forbidden frequency band," IEEE Trans. Microw. Theory Tech., vol. 47, no. 11, pp. 2059-2074, Nov. 1999.

[94] D. Sievenpiper, H.-P. Hsu, J. Schaffner, G. Tangonan, R. Garcia, and S. Ontiveros, "Low-profile, four-sector diversity antenna on high-impedance ground plane," Electron. Lett., vol. 36, pp. 1343-1345, Aug. 2000 .

[95] R. Coccioli, F.-R. Yang, K.-P. Ma, and T. Itoh, "A novel TEM waveguide using uniplanar compact photonic-bandgap (UC-PBG) structure," IEEE Trans. Microw. Theory Tech., vol. 47, no. 11, pp. 2092-2098, Nov. 1999.

[96] F.-R. Yang, K.-P. Ma, Y. Qian, and T. Itoh, "Aperture-coupled patch antenna on UC-PBG substrate," IEEE Trans. Microw. Theory Tech., vol. 47, no. 11, pp. 2123-2130, Nov. 1999.

[97] C. Caloz and T. Itoh, "A super-compact super-broadband tapered uniplanar PBG structure for microwave and millimeter wave applications," in IEEE MTT-S Int. Microwave Symp. Dig., vol. 2, Seattle, WA, Jun. 2002, pp. 1369-1372.

[98] J. McVay, N. Engheta, and A. Hoorfar, "Peano high-impedance surfaces," in Proc. URSI Int. Electromagnetic Theory Symp., vol. 1, Pisa, Italy, May 23-27, 2004, pp. 284-286.

[99] J. McVay, N. Engheta, and A. Hoorfar, "High-impedance metamaterial surfaces using Hilbert-curve inclusions," IEEE Microw. Wireless Compon. Lett., vol. 14, no. 3, pp. 130-132, Mar. 2004.

[100] A. Erentok, P. Luljak, and R. W. Ziolkowski, "Antenna performance near a volumetric metamaterial realization of an artificial magnetic conductor," IEEE Trans. Antennas Propag., vol. 53, no. 1, pp. 160-172, Jan. 2005.

[101] A. Alù, N. Engheta, and R. W. Ziolkowski, "FDTD simulation of tunneling and 'growing exponential' in a pair of epsilon-negative and mu-negative slabs," in USNC-URSI Nat. Radio Science Meeting Dig., Monterey, CA, Jun. 20-26, 2004, p. 18.
[102] A. Alù and N. Engheta, "Radiation from a traveling-wave current sheet at the interface between a conventional material and a material with negative permittivity and permeability," Microwave Opt. Technol. Lett., vol. 35, no. 6, pp. 460-463, Dec. 2002.

[103] J. Pacheco, Jr., T. M. Grzegorczyk, B.-L. Wu, Y. Zhang, and J. A. Kong, "Power propagation in homogeneous isotropic frequencydispersive left-handed media," Phys. Rev. Lett., vol. 89, Dec. 2002 Paper 257401

[104] R. W. Ziolkowski, "Gaussian beam interactions with double negative (DNG) metamaterials," in Negative Refraction Metamaterials: Fundamental Properties and Applications, G. V. Eleftheriades and K. G. Balmain, Eds., to be published.

[105] S. A. Cummer, "Dynamics of causal beam refraction in negative refractive index materials," Appl. Phys. Lett., vol. 82, pp. 2008-2010, Mar. 2003.

[106] C. L. Holloway, E. F. Kuester, J. Baker-Jarvis, and P. Kabos, "A double negative (DNG) composite medium composed of magnetodielectric spherical particles embedded in a matrix," IEEE Trans. Antennas Propag., vol. 51, no. 10, pp. 2596-2603, Oct. 2003.

[107] A. N. Lagarkov and V. N. Kisel, "Electrodynamics properties of simple bodies made of materials with negative permeability and negative permittivity," Dokl. Phys., vol. 46, no. 3, pp. 163-165, 2001.

[108] — "Electrodynamics properties of simple bodies made of materials with negative permeability and negative permittivity" (in Russian), Dokl. Akad. Nauk SSSR, vol. 377, no. 1, pp. 40-43, 2001

[109] R. A. Silin and I. P. Chepurnykh, "On media with negative dispersion," J. Commun. Technol. Electron., vol. 46, no. 10, pp. 1121-1125, 2001.

[110] - "On media with negative dispersion" (in Russian), Radiotekhnika, vol. 46, no. 10, pp. 1212-1217, 2001.

[111] M. W. Feise, P. J. Bevelacqua, and J. B. Schneider, "Effects of surface waves on behavior of perfect lenses," Phys. Rev. B, Condens. Matter, vol. 66, 2002. Paper 035113.

[112] A. Ishimaru and J. Thomas, "Transmission and focusing of a slab of negative refractive index," in Proc. URSI Nat. Radio Science Meeting, San Antonio, TX, Jul. 2002, p. 43.

[113] P. F. Loschialpo, D. L. Smith, D. W. Forester, and F. J. Rachford, "Electromagnetic waves focused by a negative-index planar lens," Phys. Rev. E, Stat. Phys. Plasmas Fluids Relat. Interdiscip. Top., vol. 67, 2003. Paper 025 602(R).

[114] M. K. Kärkkäinen, "Numerical study of wave propagation in uniaxially anisotropic Lorentzian backward-wave slabs," Phys. Rev. E, Stat. Phys. Plasmas Fluids Relat. Interdiscip. Top., vol. 68, 2003. Paper 026602.

[115] R. W. Ziolkowski and C.-Y. Cheng, "Lumped element models of double negative metamaterial-based transmission lines," Radio Sci., vol. 39, Apr. 2004. Paper RS2017.

[116] D. R. Smith and D. Schurig, "Electromagnetic wave propagation in media with indefinite permittivity and permeability tensors," Phys. Rev. Lett., vol. 90, no. 7, Feb. 2003. Paper 077405.

[117] S. Hrabar and J. Bartolic, "Backward waveguide based on uniaxial anisotropic negative permeability metamaterials," in Proc. 17th Int. Applied Electromagnetics and Communications Conf., Oct. 2003, pp. 251-254.

[118] R. Marques, J. Martel, F. Mesa, and F. Medina, "Left-handed-media simulation and transmission of EM waves in subwavelength slit-ring-resonator-loaded metallic waveguides," Phys. Rev. Lett., vol. 89, no. 18, Oct. 28, 2002. Paper 183901

[119] A. Taflove, Computational Electrodynamics: The Finite-Difference Time-Domain Method. Norwood, MA: Artech House, 1995.

[120] D. C. Wittwer and R. W. Ziolkowski, "Two time-derivative Lorentz material (2TDLM) formulation of a Maxwellian absorbing layer matched to a lossy media," IEEE Trans. Antennas Propag., vol. 48, no. 2, pp. 192-199, Feb. 2000

[121] D. C. Wittwer and R. W. Ziolkowski, "Maxwellian material based absorbing boundary conditions for lossy media in 3D," IEEE Trans. Antennas Propag., vol. 48, no. 2, pp. 200-213, Feb. 2000.

[122] D. L. Lee, Electromagnetic Principles of Integrated Optics. New York: Wiley, 1986.

[123] B. E. A. Saleh and M. C. Teich, Fundamentals of Photonics. New York: Wiley, 1991, pp. 94-95.

[124] C. F. Bohren and D. R. Huffman, Absorption and Scattering of Light by Small Particles. New York: Wiley, 1983.

[125] C. A. Balanis, Antenna Theory: Analysis and Design, 2nd ed. New York: Wiley, 1997

[126] F. Gardiol, Microstrip Circuits. New York: Wiley, 1994, pp. 48-50.

[127] W. J. Getsinger, "Microstrip dispersion model," IEEE Trans. Microw. Theory Tech., vol. MTT-21, no. 1, pp. 34-39, Jan. 1973. 


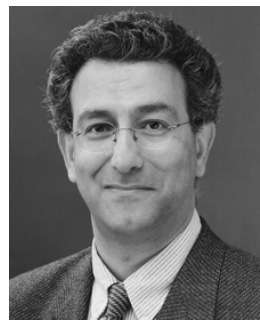

Nader Engheta (S'80-M'82-SM'89-F'96) received the B.S. degree in electrical engineering from the University of Tehran, Tehran, Iran, in 1978, and the M.S. degree in electrical engineering and Ph.D. degree in electrical engineering (with a minor in physics) from the California Institute of Technology (Caltech), Pasadena, in 1979 and 1982, respectively.

From June 1982 to June 1983, he was a Post-Doctoral Research Fellow with Caltech. From June 1983 to June 1987, he was a Senior Research Scientist with the Dikewood Division, Kaman Sciences Corporation, Santa Monica, CA. In July 1987, he joined the faculty of the University of Pennsylvania, Philadelphia, where he is currently the H. Nedwill Ramsey Professor of Electrical and Systems Engineering. He is also a member of the David Mahoney Institute of Neurological Sciences, University of Pennsylvania, and a member of the Bioengineering Graduate Group, University of Pennsylvania. $\mathrm{He}$ was the graduate group chair of electrical engineering from July 1993 to June 1997. He was an Associate Editor for Radio Science (1991-1996). He was on the Editorial Board of the Journal of Electromagnetic Waves and Applications. He has guest edited/co-edited several special issues, namely, the "Special Issue of Wave Interaction with Chiral and Complex Media" of the Journal of Electromagnetic Waves and Applications (1992), Special Issue of Antennas and Microwaves (from the 13th Annual Benjamin Franklin Symposium) of the Journal of the Franklin Institute (1995), the Special of Issue of Electrodynamics in Complex Environments of Wave Motion (2001). His research interests and activities are in the areas of fields and waves phenomena, MTMs, theory of nanooptics and nanophotonics, nanoelectromagnetism, miniaturized antennas, through-wall microwave imaging, electromagnetics/electrophysics of event-related brain cortical potentials [e.g., electroencephalography (EEG)], physics of information contents in polarization vision, bio-inspired/biomimetic sensing, processing, and displaying polarization information, reverse-engineering of polarization vision and information sensing in nature, bio-inspired hyperspectral imaging, mathematics of fractional operators, and fractal domains.

Dr. Engheta is a Guggenheim Fellow and a Fellow of the Optical Society of America. He is a member of the American Physical Society (APS), the American Association for the Advancement of Science (AAAS), Sigma Xi, Commissions B and D of the U.S. National Committee (USNC) of the International Union of Radio Science (URSI), and a member of the Electromagnetics Academy. He was the chair (1989-1991) and vice-chair (1988-1989) of the joint chapter of the IEEE Antennas and Propagation (AP)/Microwave Theory and Techniques (MTT) Philadelphia Section. He is an elected member of the Administrative Committee (AdCom) of the IEEE Antennas and Propagation Society (IEEE AP-S) since January 2003. He has organized and chaired various special sessions in international symposia. He is an associate editor for the IEEE ANTENNAS AND WIRELESS PROPAGATION LETTERS (2002-present) and was an associate editor for the IEEE TRANSACTIONS ON ANTENNA AND PROPAGATION (1996-2001). He coedited the Special Issue on Metamaterials of the IEEE TRANSACTIONS ON ANTENNAS AND PROPAGATION (2003). He was a recipient of the IEEE Third Millennium Medal. He has also been the recipient of various awards and distinctions for his scholarly research contributions and teaching activities including the UPS Foundation Distinguished Educator Term Chair (July 1999-June 2000), the Fulbright Naples Chair Award for Naples, Italy (1998), a 1989 National Science Foundation (NSF) Presidential Young Investigator (PYI) Award, two-time recipient of the S. Reid Warren, Jr. Award for distinguished teaching from the School of Engineering and Applied Science, University of Pennsylvania (1993 and 2002), the 1994 Christian F. and Mary R. Lindback Foundation Award, and the W. M. Keck Foundation's 1995 Engineering Teaching Excellence Award. He served as an IEEE Antennas and Propagation Society Distinguished Lecturer from 1997 to 1999.

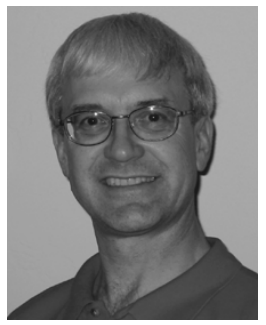

Richard W. Ziolkowski (M'87-SM'91-F'94) received the Sc.B. degree in physics (magna cum laude) (with honors) from Brown University, Providence, RI, in 1974, and the M.S. and Ph.D. degrees in physics from the University of Illinois at Urbana-Champaign, in 1975 and 1980, respectively.

From 1981 to 1990 , he was a member of the Engineering Research Division, Lawrence Livermore National Laboratory, and served as the leader of the Computational Electronics and Electromagnetics Thrust Area for the Engineering Directorate from 1984 to 1990. In 1990, he joined the Department of Electrical and Computer Engineering, University of Arizona, Tucson, as an Associate Professor, and became a Full Professor in 1996. He currently serves as the Kenneth Von Behren Chaired Professor. His research interests include the application of new mathematical and numerical methods to linear and nonlinear problems dealing with the interaction of acoustic and electromagnetic waves with complex media, MTMs, and realistic structures. For the Optical Society of America (OSA), he was a co-guest editor of the 1998 special issue of Journal of Optical Society of American A, Optical Image Science featuring mathematics and modeling in modern optics.

Prof. Ziolkowski is a member of Tau Beta Pi, Sigma Xi, Phi Kappa Phi, the American Physical Society, the Optical Society of America, the Acoustical Society of America, and Commissions B (Fields and Waves) and D (Electronics and Photonics) of URSI (International Union of Radio Science). He served as the vice chairman of the 1989 IEEE Antennas and Propagation Society (IEEE AP-S) and URSI Symposium, San Jose, CA, and as the Technical Program chairperson for the 1998 IEEE Conference on Electromagnetic Field Computation, Tucson, AZ. He served as a member of the IEEE Antennas and Propagation Society Administrative Committee (AdCom) (2000-2002). $\mathrm{He}$ was an associate editor for the IEEE TRANSACTIONS ON ANTENNAS AND PROPAGATION (1993-1998). He was a co-guest editor ( for the October 2003 IEEE TRANSACTIONS ON ANTENNAS and PROPAGATION "Special Issue on Metamaterials." He served as the IEEE AP-S vice president in 2004. He is currently serving as the IEEE AP-S president. For the U.S. URSI Society, he has served as secretary for Commission B (Fields and Waves) (1993-1996) and as chairperson of the Technical Activities Committee (1997-1999), and as secretary for Commission D (Electronics and Photonics) (2001-2002). He served as a member-at-large of the U.S. National Committee (USNC), URSI (2000-2002) and currently serves as a member of the International Commission B Technical Activities Board. He was a co-organizer of the Photonics Nanostructures Special Symposia at the 1998, 1999, and 2000 OSA Integrated Photonics Research (IPR) Topical Meetings. He served as the chair of the IPR Sub-Committee IV, Nanostructure Photonics (2001). He was a Steering Committee member for the 27th European Space Agency (ESA) Antenna Technology Workshop on Innovative Periodic Antennas: Electromagnetic Bandgap, Left-handed Materials, Fractal and Frequency Selective Surfaces, Santiago de Compostela, Spain (March 2004). He was the recipient of the 1993 Tau Beta Pi Professor of the Year Award and the 1993 and 1998 IEEE and Eta Kappa Nu Outstanding Teaching Award. 